\title{
Antiviral activity of belladonna during Japanese encephalitis virus in- fection via inhibition of microglia activation and inflammation lead- ing to neuronal cell survival
}

\author{
Swatantra Kumar ${ }^{1 \#}$, Vimal K Maurya ${ }^{1 \#}$, Russell Kabir ${ }^{2}$, Gaurav Shrivastava ${ }^{3}$, Debadatta Nayak ${ }^{4}$, Anil \\ Khurana $^{4}$, Raj K Manchanda ${ }^{4}$, Srinivasulu Gadugu ${ }^{5}$, Karuna Shanker ${ }^{6}$, Shailendra K Saxena ${ }^{1,7 \#^{*}}$ \\ ${ }^{1}$ Centre for Advanced Research (CFAR), Faculty of Medicine, King George's Medical University (KGMU), Lucknow \\ 226003, India. \\ ${ }^{2}$ School of Allied Health, Faculty of Health, Education, Medicine, and Social Care, Anglia Ruskin University, Chelmsford \\ CM1 1SQ, United Kingdom. \\ ${ }^{3}$ National Institute of Allergy and Infectious Diseases, National Institutes of Health, Rockville, Maryland 20852, USA. \\ ${ }^{4}$ CCRH, Ministry of Ayush, Janakpuri, New Delhi 110058, India. \\ ${ }^{5}$ Department of Medicine, JSPS Government Medical College, Hyderabad 500013, India. \\ ${ }^{6}$ CSIR-Central Institute of Medicinal and Aromatic Plants, Lucknow 226015, India. \\ ${ }^{7}$ CSIR-Centre for Cellular and Molecular Biology, Hyderabad 500007, India.
}

KEYWORDS: Japanese encephalitis virus, belladonna, NS3 NTPase/helicase, atropine, scopolamine, antiviral

\begin{abstract}
Japanese encephalitis virus is one of the important CNS related pathogens for which no specific treatment available.Therefore, in the present study, we have developed a hydroalcoholic formulation of belladonna (B200) and elucidated its anti-JEV activity using neuronal and microglia cell culture models. Characterization of B200 by UPLC-PDA showed the presence of $87.76 \%$ of atropine and $6.31 \%$ of scopolamine. Neuronal cell survival assay showed that $7.01 \mu \mathrm{g} / \mathrm{ml}$ was the $\mathrm{EC}_{50}$ of $\mathrm{B} 200$ which was further confirmed by reduction in necrotic cell population by and reduction in caspase 3 and 8 enzymatic activities. Antiviral effect of B200 was confirmed by reduction in

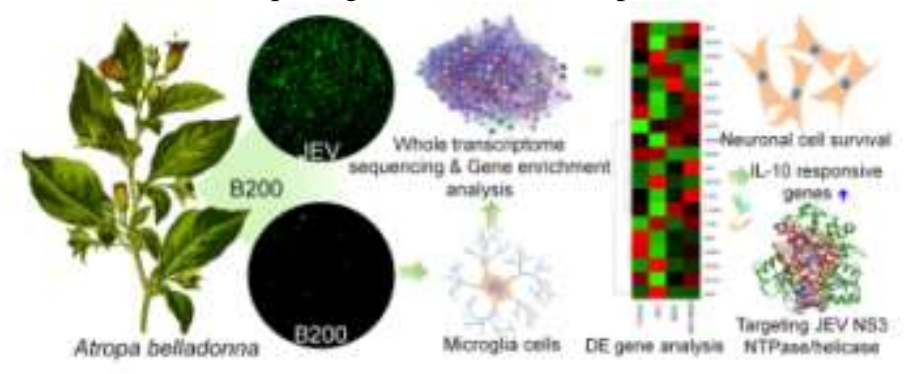
intracellular JEV level. To decipher the mechanism of antiviral, whole-transcriptome sequencing was performed, and crucial pathways involved in B200 mediated neuroprotection during JEV infection were determined. Gene expression analysis revealed that B200 reducesthe pro-apoptotic gene expression, cytokines and inflammatory gene expression observed by significant reduction in $B A D, B A X, C A S P 3, C A S P 8, I L 1 B$ and $C X C L 10$ expression and increase in IL10 responsive genes. Interestingly, our molecular docking analysis revealed that atropine and scopolamine interact with His 288 residue of NS3 protein which is crucial for RNA unwinding and ATPase activity that was further confirmed by degradation of NS3 protein. Drug likeness, ADME and toxicity analysis further suggests that atropine and scopolamine both crosses the blood brain barrier which is crucial for effective treatment of JE. These data suggest that belladonna alkaloids exhibit anti-JEV property and reduce microglia activation and inflammatory responses.
\end{abstract}

\section{INTRODUCTION}

Japanese encephalitis virus (JEV) is the prime cause of viral encephalitis in Southeast Asia with no specific treatment available. ${ }^{1} \mathrm{JEV}$ is a mosquito borne neurotropic flavivirus that belongs to family Flaviviridae. JEV is primarily transmitted by the infected mosquito bites of Culex species that circulates among pigs, water birds and humans. Incubation period of JEV infection is 5-15 days where most of the infections are asymptomatic or presented with mild symptoms. However, around 1 in 250 infections results in fatal disease which is characterized by high fever, convulsion, disorientation, seizures, coma that may results in death. ${ }^{3}$ Strikingly, the case fatality rate (CFR) of JEV infection can be high as $30 \%$ whe- 
reas $20-30 \%$ of survived individuals suffer with permanent neurological disorders. ${ }^{4} \mathrm{JEV}$ infection can be diagnosed by detection of $\operatorname{IgG}, \mathrm{IgM}$ antibodies as well as nucleic acid-based tests such as RT-PCR to perform differential diagnosis. ${ }^{5}$ There is no specific treatment available for Japanese encephalitis (JE) and current managementof the disease entirely based on symptomatic relief of the patients. However, repurposing of drugs is being investigated to identify the potential antiviral agents. ${ }^{6}$

JEV is a single stranded positive sense RNA virus of $\sim 10 \mathrm{~Kb}$ genome size which comprises of an open reading frame (ORF) that encodes for a single 3400 amino acid long polyprotein that gets cleaved into three structural protein including capsid (C), premembrane (prM) and envelope (E) and seven non-structural proteins including NS1, NS2a, NS2b, NS3, NS4a, NS4b and NS5. ${ }^{7}$ Upon infection, replication of JEV initiates in peripheral system wherein the macrophages, dendritic cells (DCs) and monocytes become primarily infected. ${ }^{8}$ The peripheral infection gets cleared off due to activation of innate immune response and therefore the level of viremia is low in the blood. ${ }^{9}$ However, JEV infects central nervous system (CNS) via breaching the blood brain barrier (BBB)which is driven by mast cells chymase. ${ }^{10}$ Neurons, astrocytes and microglia are the target cells for JEV in the brain. ${ }^{11} \mathrm{CNS}$ infection results in neuronal cell death via two mechanisms including direct wherein JEV propagation results in activation of PERK pathway ${ }^{12}$ and indirect killing where microglia activation and inflammatory response causes neuronal cell death. Microglia cells are known to act as the virus reservoir during JEV infection which gets activated and adopts inflammatory state. The activated state of microglia can be monitored by morphological transformations, cell proliferation, and increased expression of TNF- $\alpha$, IL-1 $\beta$ and RANTES. $^{13}$

JEV infection has been shown to induce programmed cell death in various cell types via IRE1/JNK pathway ${ }^{14}$ of ER stress response and Foxo signaling pathway. ${ }^{15}$ In addition, the cell death has been reported in human neural stem/progenitor cell death by increasing the GRP78. ${ }^{16}$ The helicase and protease domains of NS3 protein has been found to be one of the crucial factor that induces cellular apoptosis via inducing caspase expression. ${ }^{17}$ Several molecules have been designed to inhibit the activity of NS3 protein. However, none of them are still available for the patients use in dose dependent manner.

Belladonna is traditionally being used for the treatment of various clinical conditions due to its antiinflammatory, antimicrobial, antioxidant, anticonvulsant and analgesic properties. These diverse pharmacological properties are mainly attributed to the tropane alkaloids (atropine, scopolamine and hyoscyamine) present in roots, fruits and leaves of belladonna. ${ }^{18}$ Among all the tropane alkaloids,atropine has been found to be an antiviral against enveloped viruses. ${ }^{19} \mathrm{We}$ have recently found that belladonna is beneficial for the management of acute encephalitis syndrome (AES) including Japanese encephalitis. ${ }^{18}$ However, the role of belladonna as a direct antiviral agent has not been yet explored. Therefore, in the present study, we have developed a hydroalcoholic formulation of belladonna (B200) and elucidated its anti-JEV activity.

\section{RESULTS AND DISCUSSION}

For the first time, we have shown the direct anti-JEV effect of B200 at the molecular level. B200 treatment was found to reduce the intracellular level of JEV in both neuronal and microglia cells. To decipher the mechanism of action, we performed whole-transcriptome sequencing by generating $>14,000,000$ high quality sequences.Our gene expression analysis revealed that B200 treatment affects IL-10 responsive genes that results in suppression of microglia activation and inflammatory response that reduces neuronal cell death.We have found that B200 treatment reduces the JEV induced neuronal cell death via reducing caspase 3 and 8 enzymatic activity. Interestingly, our molecular docking analysis showed that belladonna alkaloids interact with crucial residues of NS3 protein and reduces its expression. Moreover, the drug likeness, ADME and toxicity analysis further revealed that atropine and scopolamine cross the blood brain barrier which is crucial for effective treatment of JE.

\section{Characterization of Belladonna}

The ultra-performance liquid chromatographyphotodiode array(UPLC-PDA analysis) revealed the reproducible separation of B200. The chromatogram generated by UPLC at $250 \mathrm{~nm}$ showed the peaks at 39.22 and 55.45 minutes which was confirmed by retention time matching with the reference of atropine and scopolamine respectively. Resolution of both the compounds was clear and distinct differences in the retention time made the quantification significant. B200 was found to contain $87.76 \%$ of atropine and $6.31 \%$ of scopolamine respectively (Figure 1A). In addition to atropine and scopolamine, the UPLC showed presence of other trace compounds detectable at 49.688, 58.895, 61.784 and $68.067 \mathrm{~min}$ which represented $0.49 \%, 2.62 \%, 2.41 \%$ and $0.41 \%$ of B200. However, identification of these compounds was not possible due to non-availability of the reference compounds.

The presence of these trace compounds may also have a synergistic role in antiviral mechanism mediated by B200. However, presence of higher quantity of atropine in B200 may be involved in the antiviral effects as reported in previous studies where atropine has been found beneficial against enveloped viruses. $^{19}$

\section{$\mathrm{CC}_{50}$ and $\mathrm{EC}_{50}$ of $\mathrm{B200}$}

To observe the antiviral effects of B200, first JEV infected culture supernatants were collected and viral titer was determined by plaque assay on BHK-21 cells. Ahuman microglia CHME3 and human neuroblastoma cells SHSY-5Y were treated with a range of B200 $(1-100 \mu \mathrm{g} / \mathrm{ml})$ and cell survival assay was performed to measure the $\mathrm{CC}_{50}$ of $\mathrm{B} 200$. Non-linear regression analysis suggested that $42.96 \mu \mathrm{g} / \mathrm{ml}$ and $46.73 \mu \mathrm{g} / \mathrm{mlis}$ the $\mathrm{CC}_{50}$ of $\mathrm{B} 200$ for neuronaland microglia cells respectively(Figure 1B). Since, JEV infection results in neuronal cell death. Cell survival effect of B200 during JEV infection was performed in neuronal cells infected with 5 multiplicity of infection (MOI) of JEV and treated with below $\mathrm{CC}_{50}$ of $\mathrm{B} 200$. Non-linear regression analysis suggested that $7.01 \mu \mathrm{g} / \mathrm{ml}$ is the $\mathrm{EC}_{50}$ for JEV infected cells (Figure 1C). In addition, neuronal cell survival was performed at different 
time points and percentage cell survival analysis showed that B200 treatment increases the cell survival by 1.33 fold $(p=0.0022), 1.60$ fold $(p=0.0004)$ and 2.69 fold $(p<0.0001)$ at $24 \mathrm{hrs}, 48 \mathrm{hrs}$ and $72 \mathrm{hrs}$ respectively (Figure 1D).These data demonstrate that B200 treatment reduces the JEV induced neuronal cell death.

As reported in the present study, lower doses of belladonna has been found to be beneficial in several diseased conditions and therefore may be developed as a potentialtherapeuticagents. ${ }^{18}$ The inhibition of neuronal cell death may act as one of the crucial factors for the development of ideal therapeutics for JE, since neuronal cell death is the rationale behind microglia activation and excessive release of pro-inflammatory cytokines that causes severe inflammation in the brain and death.

\section{B200 mediated neuroprotection during JEV infection}

To further observe the B200 mediated neuroprotection and cell survival during JEV infection, apoptosis assay was performed in JEV-infected B200-treated cells (Figure 2A). We have found that B200 significantly reduces the necrotic cell population and thereby increases the neuronal cell survival. Our data suggests that $2 \mu \mathrm{g} / \mathrm{ml}$ of B200 reduces percentage necrotic cell population by 1.35 fold $(p=0.0018)$, $4 \mu \mathrm{g} / \mathrm{ml}$ reduces by 2.09 fold $(\mathrm{p}=0.0001), 6 \mu \mathrm{g} / \mathrm{ml}$ reduces by 3.62 fold $(p<0.0001)$ and $8 \mu \mathrm{g} / \mathrm{ml}$ reduces by 4.67 fold $(p<0.0001)$ (Figure 2B). Caspases are the family of protease enzymes that plays a crucial role in programmed cell death. JEV infection has been shown to induce the caspase expression which causes the apoptosis in various cell types. ${ }^{20}$ To confirm this; we further determined the caspase enzymatic activity in these cells. We have found that B200 treatment significantly reduces the caspase 3 (Figure 2C) and caspase 8 (Figure 2D) activity during JEV infection.

Among all the pro-apoptotic factors, caspase 3 are the most crucial one which involve in programmed cell death via bindingwith caspase 8 to initiate the neuronal cell death. NS3 protein has been found to induce the expression of caspase 3 and cell death and therefore might be one of the crucial JEV targets for the antiviral drug development which may reduce the neuronal cell death and inhibits the viral replication process. ${ }^{17}$ In addition, caspases have been shown to inhibit the IFN mediated antiviral response via regulating cGAS-STING signaling pathway. ${ }^{21}$ Our results have shown that B200 treatment reduces the neuronal cell death via reducing the caspase 3 and 8 expression that is crucial for the development of ideal therapeutics for JE.

\section{B200 mediated reduction in intracellular level of JEV}

To elucidate the antiviral effect of B200, intracellular levels of JEV was monitored in neuronal and microglia cells by measuring the FITC expression by immunofluorescence. The level of JEV was measured at different concentration of B200 after $24 \mathrm{hrs}$ of treatment. We have found that B200 treatment significantly reduces the intracellular JEV in neuronal cells (Figure 3A). Similarly, the reduction of intracellular level of JEV was observed in microglia cells (Figure 3B). As compared with the JEV, $2 \mu \mathrm{g} / \mathrm{ml}$ of B200 treatment reduces the mean fluorescent intensity by 1.77 fold $(p=0.0002), 4 \mu \mathrm{g} / \mathrm{ml}$ reduces by 1.93 fold $(p<0.0001)$ and $6 \mu \mathrm{g} / \mathrm{ml}$ reduces by 11.19 fold $(p<0.0001)$ (Figure 3C).

As evident from the cell survival data and $\mathrm{EC}_{50}$, the reduction in intracellular JEV was approximately $50 \%$ in the cells treated with $8 \mu \mathrm{g} / \mathrm{ml}$ of $\mathrm{B} 200$.The reduction in intracellular JEV level is a direct evidence of antiviral activity of B200 as it has been recently evident in the study where screening of FDA-approved drugs for has been shown as the inhibitors of JEVinfection by monitoring the reduction of intracellular JEV level. ${ }^{22}$ These data suggest that B200 exhibits antiviral property against JEV infection.

\section{Gene enrichment and Gene Network Analysis}

To elucidate the molecular mechanism of action, we have performed whole-transcriptome sequencing by generating $>14,000,000$ high quality sequences and crucial genes involved in the antiviral effect of B200 were elucidated. To investigate the pathways involved in B200 mediated anti-JEV effect, gene enrichment and gene network analysis was performed by using Metascape which showed the involvement of several of the crucial pathways including intrinsic pathways of apoptosis, IL-10 signaling and cytokine mediated signaling pathway (Figure 4A). Molecular complex detection (MCODE) algorithm was further applied to identify the key neighborhood genes (Figure 4B). Two sets of MCODE complexes were identified one comprises of CASP8, XIAP, CASP3, GSK3B, BAD, BCL2L11 and other comprises of JAK1, SOCS5, STAT3, TYK2 and $I L 6$ (Figure $4 C$ ). Based on these data, the relative gene expression was determined based on their FPKM values.Heat map of selected gene sets was generated by Heatmapper for representing the differential gene expression in control JEV, B200 and B200+JEV where gene set (rows) and samples (columns) were hierarchically clustered based on Euclidean distance method (Figure 4D).

IL-10 responsive genes are major player for various inflammatory disease conditions and therefore can be targeted for reducing severe inflammation. ${ }^{23}$ In addition, role of these genes has been shown to be associated with JEV pathogenesis and therefore analyzing gene expressions during B200 treatment is crucial for understanding the antiviral mechanism exhibited via B200.

\section{Downregulation of pro-apoptotic genes}

Pro-apoptotic genes are the crucial players of cell death and survival. Therefore, we wished to determine the proapoptotic genes expression during B200 treatment. Caspases are the family of protease enzymes that plays a crucial role in programmed cell death. JEV infection has been shown to induce the caspases expression which causes the apoptosis in various cell types. ${ }^{20}$ Our gene expression analysis suggested that B200 treatment significantly reduces the JEV induced expression of $B A D, B A X, C A S P 3$ and $C A S P 8$ as well as increases the expression of anti-apoptotic gene XIAP. As compared with control, JEV infected cells showed 2.59fold increase $(p<0.0001)$ in $B A D$ expression which was reduced by 1.32 fold $(p=0.0025)$ upon B200 treatment (Figure 5A). JEV infected cells showed 419.69 fold increase $(p<0.0001)$ in $B A X$ expression that was reduced by 2133.86 fold $(p<0.0001)$ upon 
B200 treatment (Figure 5B). In addition, JEV infected cells showed 2.29 fold increase $(p=0.0001)$ in $C A S P 3$ expression which was reduced by B200 treatment by 1.60 fold $(p=0.0004)$ (Figure 5C). Consequently, we analyzed the CASP8 expression where JEV infected cells showed 1.38fold increase $(p=0.0015)$ in CASP8 expression when compared with control. Whereas, the B200 treatment reduces the CASP8 expression by64427.63fold $(p<0.0001)$ during JEV infection (Figure 5D). In addition, JEV infected cells showed 1.46 fold increase $(p=0.0008)$ in BCL2L11 expression which was reduced by 1.44 fold $(p=0.0009)$ upon B200 treatment (Figure $5 \mathrm{E})$.Interestingly, we have found that $\mathrm{JEV}$ infection resulted in $8.43 \mathrm{E}+11$ fold reduction $(p<0.0001)$ in XIAP expression. Whereas, the B200 treatment increases the XIAP expression by $3.71 \mathrm{E}+11$ fold $(p<0.0001)$ (Figure $5 \mathrm{~F})$. These data suggested that B200 treatment increases the microglia cell survival during JEV infection by reducing the expression of pro-apoptotic factors.

Although, JEV induced microglia cell death has not been well reported, our data suggests that JEV infection data suggests the onset of programmed cell death in microglia cells as evident from the gene expression data in consistence with the previously reported study which showed that microglia acts as a virus reservoir during JEV infection where JEV induced apoptosis was observed in mouse BV2 microglia cells. ${ }^{24}$ Our data clearly showed that B200 treatment reduces the JEV induced cell death not only in neuronal cells but also in microglia cells that eventually results in cell survival.

\section{Alteration of cytokine signaling and inflammation}

Considering the crucial role of suppressor of cytokine signaling (SOCS) family genes in inflammation and immune response exhibited by the central nervous system ${ }^{23,24}$, we planned to explore its expression in B200 mediated neuroprotection during JEV infection. Upregulation of SOCS5 has been reported during JEV infection which is responsible for inhibition of JAK-STAT signaling pathway. ${ }^{25}$ Knockdown of SOCS5 is responsible for activation of interferon (IFN) inducible anti-viral proteins and reduction of viral replication in various models. ${ }^{26} \mathrm{We}$ have found that JEV infection results in 1808.26 fold increase $(p<0.0001)$ in SOCS5expression which was found to be significantly reduced by 607.24 fold $(p<0.0001)$ upon B200 treatment (Figure 6A). The JEV NS5 protein has been shown to inhibit the type-1 IFN by blocking the nuclear translocation of $\mathrm{NF}-\kappa \mathrm{B}^{27}$ and decreases the SOCS1 and SOCS3 expression which causes increase in viral load. ${ }^{28}$ We have found that JEV infection reduces the NFKBI gene expression by3.00 fold $(p<0.0001)$ which was found to be increased by 2.30 fold $(p=0.0001)$ upon B200 treatment (Figure $6 \mathrm{~B})$. The B200 mediated translocation of NF- $\kappa \mathrm{B}$ resulted in inhibition of pro-inflammatory cytokines and activation of anti-inflammatory cytokines expression.Activated state of microglia results in overexpression of IL1B and CXCL10. We have found that, as compared with the control, JEV infected cells showed $1.02(p=0.6114)$ and 76246.5 fold increase $(p<0.0001)$ in ILIB and CXCL10 expression which was found to be downregulated by $1.24(p=0.0062)$ and 76246.5 fold $(p<0.0001)$ upon B200 treatment respectively (Figure 6C, D). In addition, JEV infected cells showed reduction in TGFB2 by
1.18 fold $(p=0.0133)$ which was increased via B200 treatment by 1.45 fold ( $p=0.0009$ ) (Figure $6 \mathrm{E}$ ). Interestingly, we have found that JEV infection resulted in downregulation of IL6expression by 1.27 fold ( $p=0.0040)$ which might be associated with the BBB permeability during early infection. We have found that B200 treatment increases the IL6 expression by 1.33 fold $(p=0.0023)$ and might involve in the inhibition of JEV entry into the CNS (Figure 6F).

Our data suggests that B200 reduces the JEV induced inflammatory state of microglia cells and thereby reduces the indirect killing of neuronal cells. Reduction in microglia mediated inflammatory response in crucial for preventing the neuronal cell death and inflammation of the brain as reported in previously developed Etanercept as a therapy for JE which reduces the inflammatory response and increases the survival.

\section{B200 mediated activation of the PI3K/AKT pathway}

PI3K/AKT pathway has been shown to play a crucial role in neuroinvasion via inducing MMP-9 expression. ${ }^{29}$ Therefore in the present study; we investigated the B200 mediated expression of PI3K/AKT expression during JEV infection. As compared with control, JEV infection increases the $P I K 3 C A$ expression by1.34 fold $(p=0.0021)$ which was found to be reduced by 1.24 fold $(p=0.0062)$ upon B200 treatment (Figure 7A). Consequently, we have found that JEV infection increases the $A K T 3$ expression by1.73 fold ( $p=0.0002)$ which was reduced by 1.27 fold $(p=0.0001)$ upon B200 treatment (Figure 7B). In addition, we investigated the expression of Foxo3 which has been found to induce apoptosis by inhibiting Foxo signaling pathway. ${ }^{15} \mathrm{We}$ have found that JEV infection reduces the FOXO3 expression by 1.42 fold $(p=0.0011)$ which has been found to increase by 1.65 fold $(p=0.0003)$ upon B200 treatment that increases the cell survival (Figure 7C). Consequently, we have determined the expression of $G S K 3 B$ owing to its crucial role in $\mathrm{BBB}$ integrity which has been found to reduce the inflammatory response $\mathrm{e}^{30}$ and negatively regulate tight junction stability of brain endothelial cells. ${ }^{31}$ We have found that JEV infection increases the $G S K 3 B$ expression by 1.22 fold $(p=0.0077)$ which was found to be reduced upon B200 treatment by 1.24 fold $(p=0.0060)$ (Figure 7D). These data suggest that B200 treatment reduces the microglia activation, inflammation and reduces the $\mathrm{BBB}$ permeability which is crucial for the prevention of JEV infection during early infection cycle.

PI3K/AKT pathways have been shown to play a crucial role in inflammatory cytokine release and therefore, reduction of inflammation via reducing these factors is crucial to prevent the fatal outcome of the disease. In addition, the prevention of infection via modulating the host immune response is also crucial for the development of effective antivirals. Our data suggests that B200 treatment may reduce the entry of the JEV during early infection cycle via modulating the BBB components as observed by decrease in GSK3Bexpression.

\section{Reduction in JEV NS3 expression}

Among all the non-structural proteins, NS3 has been shown to be one of the potential target for the development of effective antiviral agents against JEV owing to its more than 
one activity including helicase, protease, and nucleotide 5' triphosphatase (NTPase).$^{32}$ To further determine the antiviral property of B200, reduction in JEV NS3 protein expression was observed by western blotting (Figure 8A). We have found that, B200 treatment significantly reduces the NS3 protein expression after $24 \mathrm{hrs}$ of JEV infection. As compared with the JEV, $4 \mu \mathrm{g} / \mathrm{ml}$ of B200 treatment reduces the NS3 expression by 1.37 fold $(p=0.0015)$ and $6 \mu \mathrm{g} / \mathrm{ml}$ of B200 treatment reduces the NS3 expression by 2.11 fold $(p=0.0001)$ (Figure $8 \mathrm{~B})$. These data demonstrate that B200 exhibits potential antiviral activity against JEV infection.

As evident from the cell survival data and $\mathrm{EC}_{50}$ and immunofluorescence, the reduction in JEV NS3 protein expression was approximately $50 \%$ in the cells treated with $8 \mu \mathrm{g} / \mathrm{ml}$ of B200.JEV NS3 protein has been shown to induce the neuronal cell apoptosis and therefore B200 mediated reduction in the NS3 protein expression resulted in increased neuronal cell survival.

\section{Molecular docking analysis}

NS3 is a 619 amino acid long multifunctional protein of which $\mathrm{N}$-terminal exhibits serine protease activity that works under influence of a cofactor NS2b. The C-terminal is dedicated for the catalytic domain exhibiting nucleoside $5^{\prime}$ triphosphatase (NTPase)/helicase activity and 5'-terminal RNA triphosphatase activities. NS3 protein is comprises of three domains as domain 1 (171-328 aa), domain 2 (329-482 aa) and domain 3 (483-619 aa) which contains seven motifs (I, Ia, II, III, IV, V and VI). ${ }^{33}$ To confirm the antiviral activity of B200, molecular docking analysis was performed with JEV NS3 helicase/nucleoside triphosphatase. The potential binding site of the protein was predicted by metaPocket 2.0 (Figure 8C). For molecular docking, predicted binding site of NS3 protein was used. We have found that atropine and scopolamine both exhibits significant binding affinity towards catalytic domain of JEV NS3 helicase/nucleoside triphosphatase protein. We have found that atropine interacts with Arg458 and His288 residue of NS3 protein which is crucial for NTPase and RNA unwinding activity respectively (Figure 8D, E). Whereas, scopolamine was found to interact with His 288 residue at catalytic site of the NS3 protein (Figure 8F, G).

\section{B200 mediated mechanism of antiviral activity against JEV}

The ideal therapeutics for JE should focus on the various approaches considering the involvement of microglia activation and neuronal cell death. Our data suggests that the antiviral activity of B200 is multidirectional involving indirect and direct approaches. B200 mediated reduction in microglia activation was marked by decrease in inflammatory cytokines expression including $I L I B$ and $C X C L 1 O$ and therefore prevents the neuronal cell death via inflammatory cytokines. In contrasts, we have found that B200 treatment increases the antiinflammatory cytokine expression especially IL-10 responsive genes and $T G F B$ expression that further prevents the killing of neuronal cells via microglia activation. In addition, we have found that B200 treatment decreases the caspase dependent apoptosis and induces the NF- $\kappa \mathrm{B}$ translocation to the nucleus that further enhances the cell survival. Moreover, B200 treat- ment was found to reduce the PI3K/AKT signaling and increases the FOXO3 expression which leads to cell survivability. Interestingly, we have found that belladonna alkaloids atropine and scopolamine both interacts with crucial residues of NS3 NTPase/helicase protein which resulted in degradation of NS3 protein (Figure 9).

\section{B200 alkaloids crosses the BBB}

Atropine and scopolamine were found to be an orally active compound which can cross the BBB. The cLogP of atropine and scopolamine was 2.03 and 1.35 respectively, indicating that compounds are fit to be orally active compounds. Both the compounds were found to cross the $\mathrm{BBB}$ which is crucial for the treatment of JE patients. Other parameters such as absorption in intestine, distribution, metabolism and toxicity were compared and listed in Table 1.

\section{METHODS}

\section{Virus and cells}

We have used JEV Gp05 strain (NCBI accession no: FJ979830) which was isolated from human brain during an epidemic of Japanese encephalitis (JE) in 2005 in Gorakhpur, U.P. India. ${ }^{34} \mathrm{JEV}$ was propagated in baby hamster kidney cells (BHK-21) cells, which was cultured in 1x DMEM, 10\% fetal bovine serum (FBS) to generate the stock of JEV. To determine the virus titer in culture supernatant, plaque assay was performed and multiplicity of infection (MOI) was determined. To study the impact of B200 on neuronal cells, human neuroblastoma (SH-SY5Y) cells were cultured in 1X DMEMF12with $10 \%$ FBS and 2 g/L of sodium bicarbonate. In addition, human microglia cell line (CHME3) was used to decipher the antiviral mechanism exhibited by B200. CHME3 cells were cultured in 1x DMEM-F12 medium with $10 \%$ FBS and 2 $\mathrm{g} / \mathrm{L}$ of sodium bicarbonate. All the cells were cultured in $0.1 \mathrm{mg} / \mathrm{ml}$ of penicillin; $0.2 \mathrm{mg} / \mathrm{ml}$ of streptomycin.

\section{Plaque assay}

To determine the virus titer, BHK-21 cells were seeded in Nunc ${ }^{\mathrm{TM}} 6$ well Cell-Culture plate with the density of $0.8 \times 10^{6}$ cells per well. Stock virus supernatant was serially diluted in chilled DMEM medium, and $0.2 \mathrm{ml}$ of diluted inoculums was used to infect the cell monolayer. After incubation of $90 \mathrm{~min}$, the cell monolayer was washed and overlay medium was added for plaque formation. Overlay medium comprises of $0.2 \%$ carboxymethyl cellulose (CMC) and DMEM medium with $2 \%$ FBS. After 3-4 days of incubation at $37^{\circ} \mathrm{C}$ in $5 \% \mathrm{CO} 2 .^{35}$ Plaque forming units (PFUs) were counted and multiplicity of infection (MOI) which was used for infection/number of cells. In the present study, MOI of 5 was used throughout the study to elucidate the antiviral property of belladonna.

\section{JEV infection ofneuronal and microglia cells}

SHSY-5Y/CHME3 cells were seeded in 6 well cell culture plates with the density of $0.3 \times 10^{6}$ cells per well. Prior to infection, cells were washed with $1 \mathrm{X}$ PBS and $0.2 \mathrm{ml}$ of inoculums (MOI of 5) was added to respective wells and incubated for $90 \mathrm{~min}$ at $37^{\circ} \mathrm{C}$ for internalization. After incubation, 
cell monolayers were washed with $1 \mathrm{x}$ PBS twice and $1 \mathrm{X}$ DMEM-F12 media with $10 \%$ FBS was added.

\section{Preparation of drugs}

Belladonna tincture was used to prepare the different dilutions of B200. Belladonna tincture, an ethanolic extract of Atropa belladonna, was prepared as per the Pharmacopoeia of India, 1971. Briefly, to prepare the $100 \mathrm{ml}$ of hydroalcoholic stock solution, whole plant extract of $10 \mathrm{gm}$ was dissolved in $100 \mathrm{ml}$ of $47 \%$ ethanol. To achieve desired concentrations of B200, stock was further serially diluted in same percentage of ethanol with high pressure homogenization. Similarly, placebo, a hydro-alcoholic solution, was prepared following the same procedure, but without belladonna tincture.

\section{Characterization of Belladonna Formulation by UPLC}

Quantitative characterization of B200 was performed by UPLC-PDA method. ${ }^{36}$ Briefly, before injection, B200 and mobile phases were filtered via $0.22 \mu \mathrm{m}$ PVDF membrane. The optimum separation was obtained with gradient mobile phase with solvent $\mathrm{A}$ (water $+0.1 \%$ formic acid) and solvent $\mathrm{B}$ (methanol). Three microliters of the B200 was injected using autosampler and total run time was 75 minutes at $0.30 \mathrm{ml} / \mathrm{min}$ flow rate. The linear gradient conditions were $0-20 \mathrm{~min}, 80 \% \mathrm{~A}$ and $20 \% \mathrm{~B} ; 20-40 \mathrm{~min}, 60 \% \mathrm{~A}$ and $40 \% \mathrm{~B} ; 40-66 \mathrm{~min}, 100 \% \mathrm{~B}$, 66-73 min, $40 \%$ A and 60\% B. PDA Detector Type UPLC LG was set at $500 \mathrm{~nm}$ and was used to quantify the optimum chromatographic signal response.

\section{Cell survival assay, CC50 and EC50}

SHSY-5Y cells were seeded with $0.01 \times 10^{6}$ cells/ well in 96 well cell culture plate. To elucidate the antiviral effect of B200, firstly median cytotoxic concentration $\left(\mathrm{CC}_{50}\right)$ was determined $24 \mathrm{hrs}$ post treatment upon treatment with range of B200 concentration $(1-100 \mu \mathrm{g} / \mathrm{ml})$. To determine the median effective concentration (EC50), cells were first infected with $5 \mathrm{MOI}$ of JEV and treated with below $\mathrm{CC}_{50}$ concentration of B200 after 24 hrs of incubation. Further, EC50 was used to measure the cell viability at different time points. Both $\mathrm{CC}_{50}$ and $\mathrm{EC}_{50}$ were measured based by cell survival assay using $50 \mathrm{~mL}$ of a $5 \mathrm{mg} / \mathrm{mL} 3$-(4,5-dimethylthiazol-2-yl)2,5-diphenyl tetrazolium bromide solution (MTT; DuchefaBiochemie, the Netherlands). Cell viability was measured as described before.

\section{Caspase 3 and 8 enzymatic activities}

Considering the crucial role of caspases in neuronal cell death and survival, caspase 3 and caspase 8 activities were measured in JEV infected B200 treated neuronal cells by using Caspase 3 assay kit (Sigma, USA) and Caspase 8 assay kit (Sigma, USA) respectively. These assays are based on the hydrolysis of Ac-DEVD-AMC by caspase 3 and Ac-IETDpNA by caspase 8 which resulted in the release of AMC and p-NA respectively which can be detected by optical absorbance at $405 \mathrm{~nm}$ and $505 \mathrm{~nm}$ respectively.

\section{Intracellular JEV levels by immunofluorescence}

To perform the immunofluroscence, SHSY-5Y cells were cultured in 4 well Lab-Tek ${ }^{\circledR}$ Chamber Slide ${ }^{\mathrm{TM}}$ with the density of $0.015 \times 10^{6}$ cells per well. Cells were infected with 1 MOI of JEV and treated with EC50 of B200. After $24 \mathrm{hrs}$ of incubation, cells were stained with anti-envelope JEV antibody followed by FITC labeled-secondary antibody (Life Technologies, USA). Fluorescence microscopy was performed using Nikon upright fluorescent microscope. The intensity of fluorescence signals was determined using fluorescence in image $\mathrm{J}$ (Fiji) software.

\section{Western blotting}

Cells were cultured, infected and treated with EC50 of B200 as mentioned above. To prepare the whole cell lysate, $2 \times$ Laemmli buffer was used and $10 \%$ separating gel was used to separate the protein by SDS-PAGE. The proteins were transferred from gel to Hybond ${ }^{\circledR}$ ECL ${ }^{\mathrm{TM}}$ Hybond ECL Nitrocellulose Membrane (Amersham, UK) using Trans-Blot Turbo (Bio-RAD, USA). The membrane was blocked for $1 \mathrm{hr}$ with $5 \%$ of BSA. Membrane was incubated with anti-envelope JEV antibody $(1: 1000)$ was used overnight at $4{ }^{\circ} \mathrm{C}$ to allow the antibodies to bind with JEV envelope protein. Striping of the membrane was performed using mild stripping buffer. The membrane was again blocked and incubated with GAPDH antibody (1:1000) (Santa Cruz Biotechnology, Inc., USA) as the control. Sheep anti-mouse IgG-HRP-conjugated secondary antibody (GE Healthcare, USA) was used to observe the protein expression using ECL kit (GE Healthcare, USA).

\section{Molecular docking}

The PDB structure of JEV NS3 protein (PDB ID: 2Z83) was retrieved from RCSB PDB databank. The potential binding site of NS3 protein was determined by using metaPocket 2.0. The ligands atropine (PubChem CID: 174174) and scopolamine (PubChem CID: 3000322) were downloaded in SDF format from PubChem database. Molecular docking was performed using Molegro Virtual Docker (MVD) and MolDock scores were compared. ${ }^{37}$ The molecular interactions (2D and 3D) were visualized by Discovery Studio 2016 client.

\section{RNA Isolation, Library Preparation and Clustering}

To decipher the pathways involved in B200 mediated antiviral property, total RNA was isolated from JEV-infected and B200 treated cells using SV total RNA systems. A total of $1000 \mathrm{ng}$ of RNA quantified by Nanodrop and RNA integrity number of 9.0 measured by 2100 Bioanalyzer Systems (Agilent, USA). Random hexamers were used for first strand cDNA synthesis using Superscript II Reverse Transcriptase following the second strand synthesis using DNA polymerase I and RNase H. The cDNAs were cleaned by Beckman Coulter AgencourtAMpure XP SPRI beads and $300 \mathrm{ng}$ of each samples was used for whole-transcriptome next-generation sequencing using TruSeq RNA Library Prep Kits for Illumina ${ }^{\circledR}$ (NEB, USA) following addition of index codes to attribute sequences for each sample. Clustering of the indexcoded samples was performed by TruSeq PE Cluster Kit v3cBot-HS (Illumina) using cBot Cluster Generation system. 
RNA-Sequencing and Analysis

Paired-end reads of 100 base pairs was generated using Illumina Hi-Seq 2500 sequencing system which was further aligned with the reference genome Homo sapiens GRCh38 by TopHat. These aligned files were used for assembling and estimation of transcripts and their abundance using Cufflinks package (v 2.2.1). ${ }^{38}$ Cuffmerge was used to combine the assembled transcripts for generation of master transcriptome and Cuffdiff was used for differential gene expression analysis. $^{39}$ Differentially expressed transcripts between JEV infected and B200 treated cells were identified using Cuffdiff data analysis pipeline where fold-change threshold of absolute fold-change $\geq 1.5$ and a statistically significant Student's t-test $\mathrm{P}$ value threshold adjusted for false discovery rate of less than 0.001 . The sequencing datasets used in the present study was $\mathrm{N}=1$. Therefore, blind dispersion method was applied to treat all samples in the experiments as replicates of the single global condition and thereby build one model for differential gene expression analysis ${ }^{40,41}$ and the $p$ value were determined using Student's t-test with Benjamini-Hochberg FDR test. We have considered the expression of transcripts with $\log 2$ fold change $\geq 1.5$ as upregulated and $\log 2$ fold change $\leq 1.5$ as downregulated. Consequently, normalized Fragments Per Kilobase of transcript per Million mapped reads (FPKM) values calculated by Cufflinks was used for downstream analysis. ${ }^{38}$ The genes involved in the B200 mediated neuroprotection during JEV infection were identified by Gene Ontology (GO) database. Metascape was used for enrichment analysis and gene network analysis. In addition, MCODE algorithm was applied to the network for identification of densely connected neighborhood genes.

\section{IL-10 measurement by RT-PCR}

To validate the expression level of IL 10, gene expression analysis was performed by real-time PCR (RT-PCR). Cells were treated as mentioned above and total RNA was isolated using TRIzol. Approximately, 200ng of isolated RNA was used to perform the cDNA synthesis using Reverse Transcription System (Promega, USA). Endpoint PCR was performed for IL 10 specific gene where GAPDH was used as the control. The amplified products were run on the $0.8 \%$ agarose gel.

\section{Drug likeness, ADME and toxicity}

High-quality drug molecules should not only exhibit specificity against the targeted proteins, but also exhibits appropriate ADMET properties. Therefore, the drug likeness of atropine and scopolamine was analyzed by Lipinski filter and the properties of alkaloids with respect to ADME and toxicity was evaluated by admetSAR. ${ }^{42}$ Considering the CNS involvement of JEV, BBB permeability were also determined for both the compounds.

\section{Statistical analysis}

The statistical difference between more than two independent groups was performed by one-way analysis of variance. GraphPad Prism was used to measure the $\mathrm{CC}_{50}$ and
$\mathrm{EC}_{50}$ of the $\mathrm{B} 200$ by non-linear regression analysis. All the experiments were performed in triplicates and the values are represented as mean \pm standard error of mean. Significance level between JEV and B200-treated cells was represented as $* * *, * *$ and $*$ for $p<0.001, p<0.01$ and $p<0.05$, respectively.

\section{ASSOCIATED CONTENT}

\section{Data Availability Statement}

The high-throughput RNA seq data is available from the GEO repository of the National Center for Biotechnology Information (NCBI), accession numberGSE1578980ther data relevant to the study's conclusions are available on request from the corresponding author.

\section{ABBREVIATIONS}

JEV, Japanese encephalitis virus; WHO, World Health Organization; B200, Belladonna formulation; CFR, Case fatality rate; JE, Japanese encephalitis; ORF, Open reading frame; DCs, Dendritic cells; CNS, Central nervous system; BBB, Blood brain barrier; UPLC-PDA, Ultra-performance liquid chromatography-photodiode array; MOI, Multiplicity of infection; NTPase,Nucleotide 5' triphosphatase; MCODE, Molecular complex detection; SOCS, Suppressor of cytokine signaling; IFN, interferon; BHK-21, Baby hamster kidney; CHME3, Human microglial clone 3 cell line, CMC, Carboxymethyl cellulose; PFU, Plaque forming units; CC50, Median cytotoxic concentration; EC50, Median effective concentration; MTT, 3-(4,5-dimethylthiazol-2-yl)-2,5-diphenyl tetrazolium bromide; RIN, RNA integrity number; FPKM, Fragments per kilobase of transcript per million mapped reads;GO, Gene Ontology

\section{AUTHORS INFROMATION}

\section{Corresponding Author}

Prof. Shailendra K Saxena

Department of Centre for Advanced Research (CFAR), Faculty of Medicine, King George's Medical University (KGMU), Lucknow, India, Tel: 91522 2257450; Fax: 91522 2257450;

E-mail: shailen@kgmcindia.edu

\section{Author Contributions}

SKS conceived the idea. SK and SKS collected the data, devised the initial draft and reviewed the final draft. SKS, SK, VKM, RK, GS, DN, AK, RKM, SG, and KS finalized the draft for submission. All authors read and approved the final version of the manuscript.

\section{ACKNOWLEDGMENTS}

The authors are grateful to the Vice Chancellor, King George's Medical University (KGMU), Lucknow, India for the encouragement for this work.

\section{Funding Sources}

SK Saxena is supported by CCRH, Government of India. The authors have no other relevant affiliations or financial involvement with any organization or entity with a finan- 
cial interest in or financial conflict with the subject matter or materials discussed in the manuscript apart from those disclosed.

\section{Conflict of Interest}

The authors declare no competing financial interest. The research was conducted in the absence of any commercial or financial relationships that could be construed as a potential conflict of interest.

\section{REFERENCES}

1. Saxena, S. K., Kumar, S., and Maurya, V. K. (2019) Pathogen-associated acute encephalitis syndrome: therapeutics and management. Future Microbiol. 14, 259-262.

2. Saxena, S. K., Kumar, S., and Haikerwal, A. (2020) Animal flaviviruses, in: Malik, Y.S., Singh, R. K., Yadav, M. P. (Eds.), Emerging and transboundary animal viruses. Springer, Singapore. pp. 137-159.

3. World Health Organization, (2019) Japanese encephalitis. https://www.who.int/news-room/factsheets/detail/japanese-encephalitis (accessed 09 August 2020).

4. Sarkari, N. B., Thacker, A. K., Barthwal, S. P., Mishra, V. K., Prapann, S., Srivastava, D., and Sarkari, M. (2012) Japanese encephalitis (JE) part II: 14 years' follow-up of survivors. J Neurol. 259, 58-69.

5. Saxena, S. K., Kumar, S., Maurya, V. K., and Bhatt, M. L. (2019) The global distribution and burden of dengue and Japanese encephalitis co-infection in acute encephalitis syndrome, in: Rodriguez-Morales, A. J. (Ed.), Current topics in neglected tropical diseases.IntechOpen. https://doi.org/10.5772/intechopen.89792.

6. Turtle, L., and Solomon, T. (2018) Japanese encephalitis the prospects for new treatments. Nat Rev Neurol. 14, 298 313.

7. Schuh, A. J., Tesh, R. B., and Barrett, A. D. (2011) Genetic characterization of Japanese encephalitis virus genotype II strains isolated from 1951 to 1978. J Gen Virol. 92, 516527.

8. Wang, P., Hu, K., Luo, S., Zhang, M., Deng, X., Li, C., Jin, W., Hu, B., He, S., Li, M., Du, T., Xiao, G., Zhang, B., Liu, Y., and $\mathrm{Hu}, \mathrm{Q}$. (2016) DC-SIGN as an attachment factor mediates Japanese encephalitis virus infection of human dendritic cells via interaction with a single high-mannose residue of viral E glycoprotein. Virology. 488, 108-119.

9. Solomon T. (2004) Flavivirus encephalitis. N Engl J Med. 351, 370-378

10. Hsieh, J. T., Rathore, A., Soundarajan, G., \& St John, A. L. (2019) Japanese encephalitis virus neuropenetrance is driven by mast cell chymase. Nat Commun. 10, 706.

11. Myint, K. S., Kipar, A., Jarman, R. G., Gibbons, R. V., Perng, G. C., Flanagan, B., Mongkolsirichaikul, D., Van Gessel, Y., \& Solomon, T. (2014) Neuropathogenesis of Japanese encephalitis in a primate model. PLoSNegl Trop Dis. 8, e2980.

12. Wang, Q., Xin, X., Wang, T., Wan, J., Ou, Y., Yang, Z., Yu, Q., Zhu, L., Guo, Y., Wu, Y., Ding, Z., Zhang, Y., Pan, Z., Tang, Y., Li, S., and Kong, L. (2019) Japanese Encephalitis Virus Induces Apoptosis and Encephalitis by Activating the PERK Pathway. J Virol. 93, e00887-19.

13. Chen, C. J., Ou, Y. C., Lin, S. Y., Raung, S. L., Liao, S. L., Lai, C. Y., Chen, S. Y., and Chen, J. H. (2010) Glial activation involvement in neuronal death by Japanese encephalitis virus infection. J Gen Virol. 91, 1028-1037.
14. Huang, M., Xu, A., Wu, X., Zhang, Y., Guo, Y., Guo, F., Pan, Z., and Kong, L. (2016) Japanese encephalitis virus induces apoptosis by the IRE1/JNK pathway of ER stress response in BHK-21 cells. Arch Virol. 161, 699-703.

15. Guo, F., Yu, X., Xu, A., Xu, J., Wang, Q., Guo, Y., Wu, X., Tang, Y., Ding, Z., Zhang, Y., Gong, T., Pan, Z., Li, S., and Kong, L. (2018) Japanese encephalitis virus induces apoptosis by inhibiting Foxo signaling pathway. Vet Microbiol. 220, 73-82.

16. Mukherjee, S., Singh, N., Sengupta, N., Fatima, M., Seth, P., Mahadevan, A., Shankar, S. K., Bhattacharyya, A., and Basu, A. (2017) Japanese encephalitis virus induces human neural stem/progenitor cell death by elevating GRP78, PHB and hnRNPC through ER stress. Cell Death Dis. 8, e2556.

17. Yiang, G. T., Chen, Y. H., Chou, P. L., Chang, W. J., Wei, C. W., Yu, and Y. L. (2013) The NS3 protease and helicase domains of Japanese encephalitis virus trigger cell death via caspasedependent and independent pathways. Mol Med Rep. 7, 826-830.

18. Maurya, V. K., Kumar, S., Kabir, R., Shrivastava, G., Shanker, K., Nayak, D., Khurana, A., Manchanda, R. K., Gadugu, S., Kar, S. K., Verma, A. K., and Saxena, S. K. (2020). Dark Classics in Chemical Neuroscience: An Evidence-Based Systematic Review of Belladonna. ACS Chem Neurosci. 10.1021/acschemneuro.0c00413. Advance online publication. Doi:10.1021/acschemneuro.0c00413

19. Yamazaki, Z., and Tagaya, I. (1980) Antiviral effects of atropine and caffeine. J Gen Virol. 50, 429-431.

20. Tsao, C. H., Su, H. L., Lin, Y. L., Yu, H. P., Kuo, S. M., Shen, C. I., Chen, C. W., and Liao, C. L. (2008) Japanese encephalitis virus infection activates caspase- 8 and -9 in a FADD-independent and mitochondrion-dependent manner. J Gen Virol. 89, 1930-1941.

21. Baker, B. J., Akhtar, L. N., and Benveniste, E. N (2009) SOCS1 and SOCS3 in the control of CNS immunity. Trends Immunol. 30, 392-400.

22. Yakass, M. B., Franco, D., and Quaye, O. (2020) Suppressors of Cytokine Signaling and Protein Inhibitors of Activated Signal Transducer and Activator of Transcriptions As Therapeutic Targets in Flavivirus Infections. J Interferon Cytokine Res. 40, 1-18.

23. Sharma, N., Kumawat, K. L., Rastogi, M., Basu, A., \& Singh, S. K. (2016). Japanese Encephalitis Virus exploits the microRNA-432 to regulate the expression of Suppressor of Cytokine Signaling (SOCS) 5. Scientific reports, 6, 27685.

24. Zhang, J., Li, Z., Huang, J., Yin, H., Tian, J., and Qu, L. (2019) miR-26a Inhibits Feline Herpesvirus 1 Replication by Targeting SOCS5 and Promoting Type I Interferon Signaling. Viruses.12, 2

25. Ye, J., Chen, Z., Li, Y., Zhao, Z., He, W., Zohaib, A. Song, Y., Deng, C., Zhang, B., Chen, H., and Cao, S. (2017) Japanese Encephalitis Virus NS5 Inhibits Type I Interferon (IFN) Production by Blocking the Nuclear Translocation of IFN Regulatory Factor 3 and NF-кB. $J$ Virol. 91(8), e00039-17.

26. Kundu, K., Dutta, K., Nazmi, A., and Basu, A. (2013) Japanese encephalitis virus infection modulates the expression of suppressors of cytokine signaling (SOCS) in macrophages: implications for the hosts' innate immune response. Cell Immunol. 285, 100-110.

27. Yang, C. M., Lin, C. C., Lee, I. T., Lin, Y. H., Yang, C. M., Chen, W. J., Jou, M. J., and Hsiao, L. D. (2012) Japanese encephalitis virus induces matrix metalloproteinase-9 expression via a ROS/c-Src/PDGFR/PI3K/Akt/MAPKsdependent AP-1 pathway in rat brain astrocytes. J Neuroinflammation. 9, 12 . 
28. Ramirez, S. H., Fan, S., Zhang, M., Papugani, A., Reichenbach, N., Dykstra, H., Mercer, A. J., Tuma, R. F., \&Persidsky, Y. (2010). Inhibition of glycogen synthase kinase 3beta (GSK3beta) decreases inflammatory responses in brain endothelial cells. Am J Pathol. 176, 881-892.

29. Ramirez, S. H., Fan, S., Dykstra, H., Rom, S., Mercer, A., Reichenbach, N. L., Gofman, L., and Persidsky, Y. (2013) Inhibition of glycogen synthase kinase $3 \beta$ promotes tight junction stability in brain endothelial cells by half-life extension of occludin and claudin-5. PLoS One. 8, e55972.

30. Luo, D., Vasudevan, S. G., and Lescar, J. (2015) The flavivirus NS2B-NS3 protease-helicase as a target for antiviral drug development. Antiviral Res. 118, 148-158.

31. Yamashita, T., Unno, H., Mori, Y., Tani, H., Moriishi, K., Takamizawa, A., Agoh, M., Tsukihara, T., and Matsuura, Y. (2008) Crystal structure of the catalytic domain of Japanese encephalitis virus NS3 helicase/nucleoside triphosphatase at a resolution of 1.8A. Virology. 373, 426-436.

32. Mishra, N., and Saxena, S.K. (2009) Secondary structure analysis of $3^{\prime}$ NCR of JEV for similarity and differences: implication for virulence, attenuation and vaccine development. https://www.ncbi.nlm.nih.gov/nuccore/254596857

33. Singh, S., Sharma, M., Kumar, S., and Gowal, D. (2015) Standardization of serum neutralization assay of Japanese encephalitis virus (Nakayama NIH strain) on BHK-21 (Cl13) cell line. Acta Virol. 59, 234-239.

34. Srivastava, M., Singh, M., Maurya, P., Srivastava, N., Gupta, N., and Shanker, K. (2019) Simultaneous quantification of five bioactive phenylethanoid, iridoid, and flavonol glycosides in Durantaerecta L.: Ultra performance liquid chromatography method validation and uncertainty measurement. J Pharm Biomed Anal. 174,711-717.

35. Thomsen, R., and Christensen, M. H. (2006) MolDock: a new technique for high-accuracy molecular docking. $J \mathrm{Med}$ Chem. 49, 3315-3321.

36. Trapnell, C., Roberts, A., Goff, L., Pertea, G., Kim, D., Kelley, D.R., Pimentel, H., Salzberg, S.L., Rinn, J.L., and Pachter, L. (2012) Differential gene and transcript expression analysis of RNA-seq experiments with TopHat and Cufflinks. Nat Protoc. 7, 562-578.

37. Seyednasrollah. F., Laiho, A., and Elo, L.L. (2015) Comparison of software packages for detecting differential expression in RNA-seq studies. Brief Bioinform. 16, 59-70.

38. Bukosza, E.N., Kratochwill, K., Kornauth, C., Schachner, H., Aufricht, C., and Gebeshuber, C.A. (2020) Podocyte RNA sequencing reveals Wnt- and ECM-associated genes as central in FSGS. PLoS One. 15, e0231898.

39. Galipon, J., Ishii, R., Suzuki, Y., Tomita, M., and Ui-Tei, K. (2017) Differential Binding of Three Major Human ADAR Isoforms to Coding and Long Non-Coding Transcripts. Genes (Basel). 8, pii: E68.

40. Yang, H., Lou, C., Sun, L., Li, J., Cai, Y., Wang, Z., Li, W., Liu, G., and Tang, Y. (2019). admetSAR 2.0: webservice for prediction and optimization of chemical ADMET properties. Bioinformatics. 35, 1067-1069. 

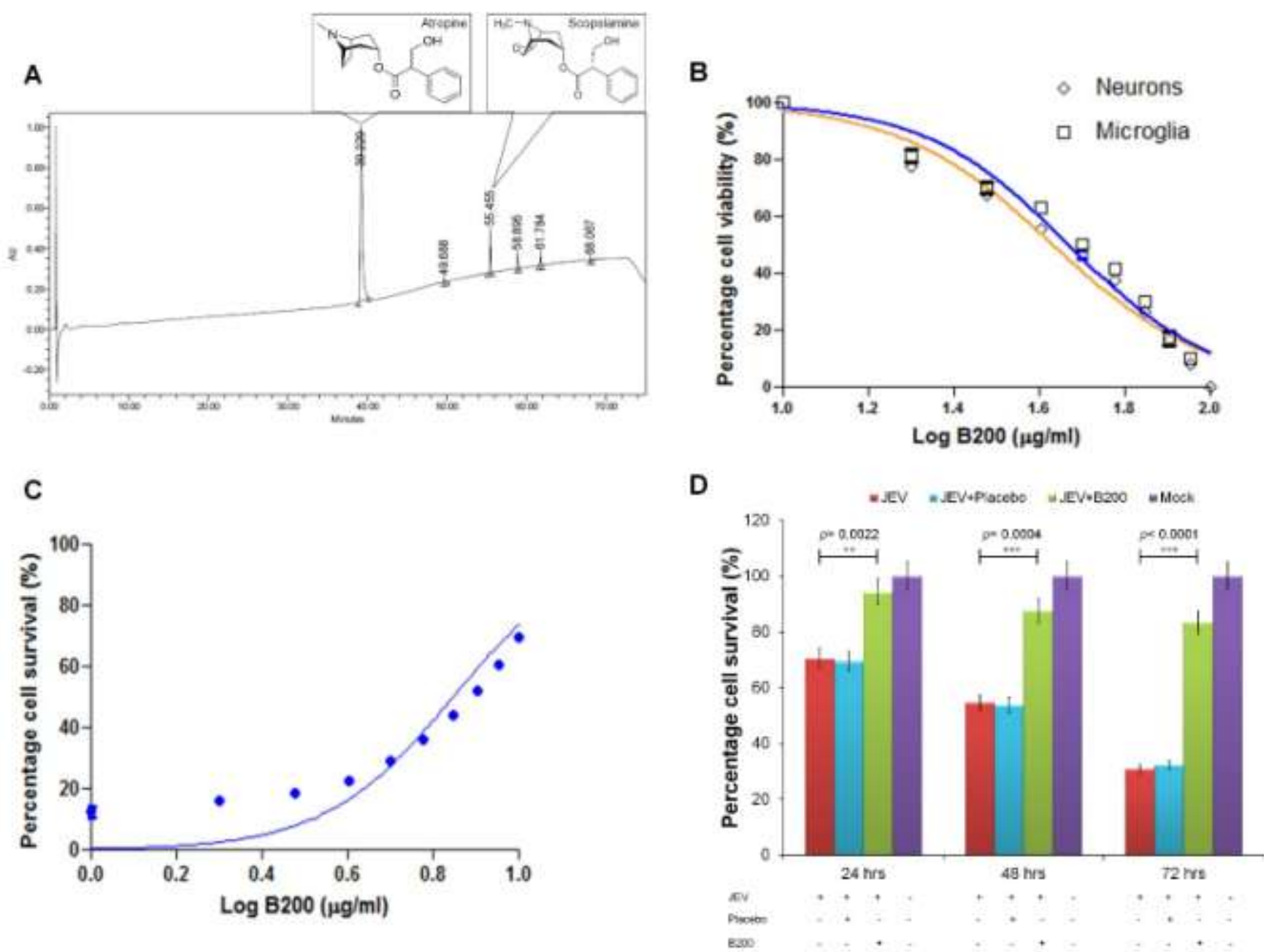

Figure 1: Characterization of belladonna formulation (B200) and its effective concentration against JEV.(A) Quantitative determination of B200 was performed by UPLC at $250 \mathrm{~nm}$ showing the peaks of possible compounds. B200 was found to contain $87.76 \%$ of atropine (39.22) and $6.31 \%$ of scopolamine (55.45). (B)Cells treated with (1-100 $\mu \mathrm{g} / \mathrm{ml})$ of B200 and the percentage cell viability curve with $42.88 \mu \mathrm{g} / \mathrm{ml}$ is the $\mathrm{CC}_{50}$. $(\mathbf{C})$ Cells infected with $\mathrm{JEV}$ and treated with below $\mathrm{CC}_{50}$ of $\mathrm{B}^{200}$ and percentage cell survival curve showing that $7.01 \mu \mathrm{g} / \mathrm{ml}$ is the $\mathrm{EC}_{50}$. (D) Cells were treated with $\mathrm{EC}_{50}$ of B200 during JEV infection and percentage cell survival at different time point showing that B200 treatment increases the cell survival by 1.33 fold ( $p=0.0022$ ), 1.60 fold $(p=0.0004)$ and 2.69 fold $(p<0.0001)$ at $24 \mathrm{hrs}, 48 \mathrm{hrs}$ and $72 \mathrm{hrs}$ respectively. The experiments were carried out in triplicates and the values are represented as $\mathrm{AM} \pm \mathrm{SD}$. 
A

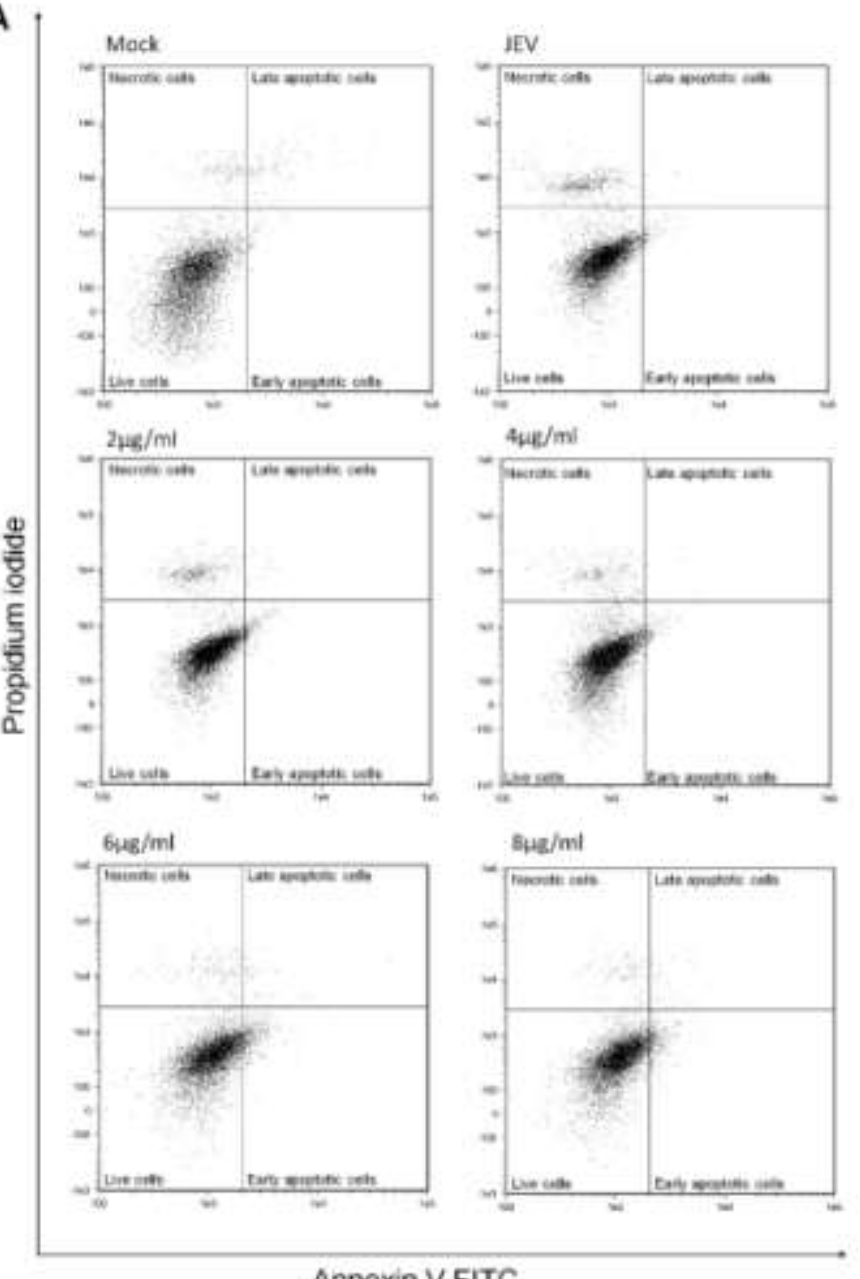

B

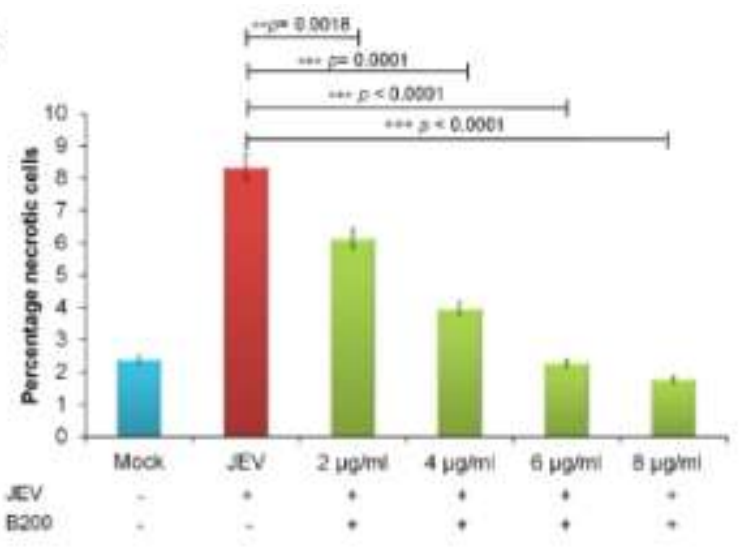

C
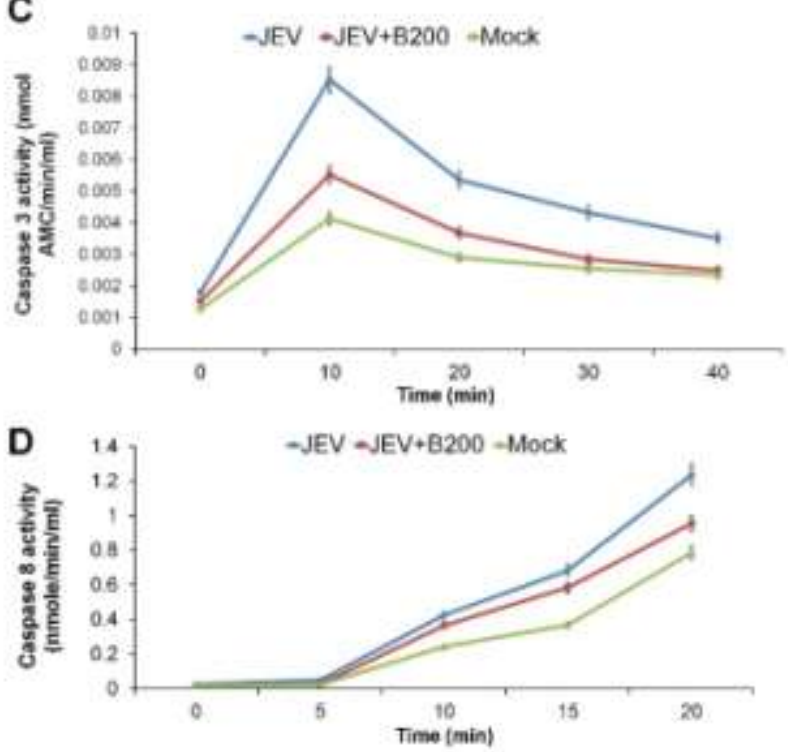

Figure 2: B200 reduces apoptosis in JEV infected cells. (A) Flow cytometry of Annexin-V-FITC and propidium iodide stained cells showing the gradual reduction in necrotic cell population in B200 treated cells during JEV infection.(B) Graph showing that $2 \mu \mathrm{g} / \mathrm{ml}$ of B200 reduces percentage necrotic cell population by 1.35 fold $(p=0.0018), 4 \mu \mathrm{g} / \mathrm{ml}$ reduces by 2.09 fold $(p=0.0001)$, $6 \mu \mathrm{g} / \mathrm{ml}$ reduces by 3.62 fold $(p<0.0001)$ and $8 \mu \mathrm{g} / \mathrm{ml}$ reduces by 4.67 fold $(p<0.0001)$. (C) Graph showing significant reduction in caspase 3 activity in JEV infected B200 treated cells. (D) Graph showing significant reduction in caspase 8 activity in JEV infected B200 treated cells. The data is expressed as the mean \pm standard error of mean $(n=3)$ where $p$ values are represented as the difference between JEV-infected cells and JEV-infected B200-treated cells. 
A
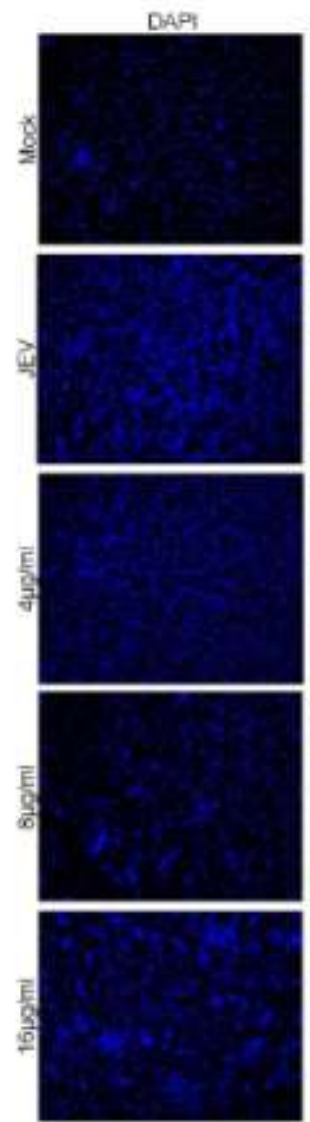

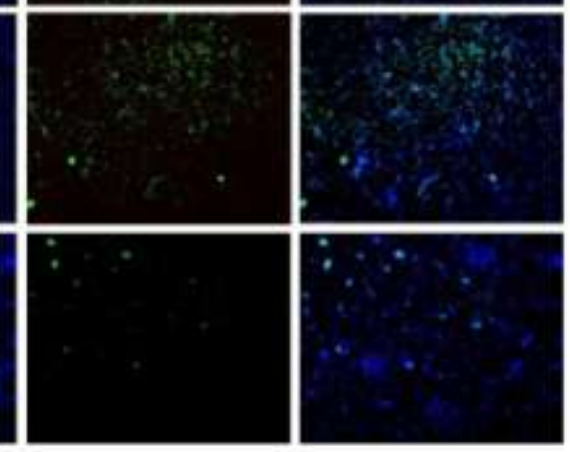

FITC
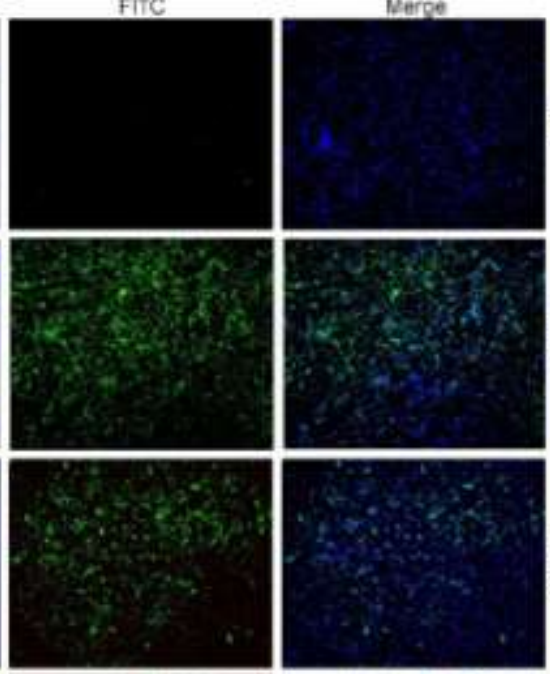

B
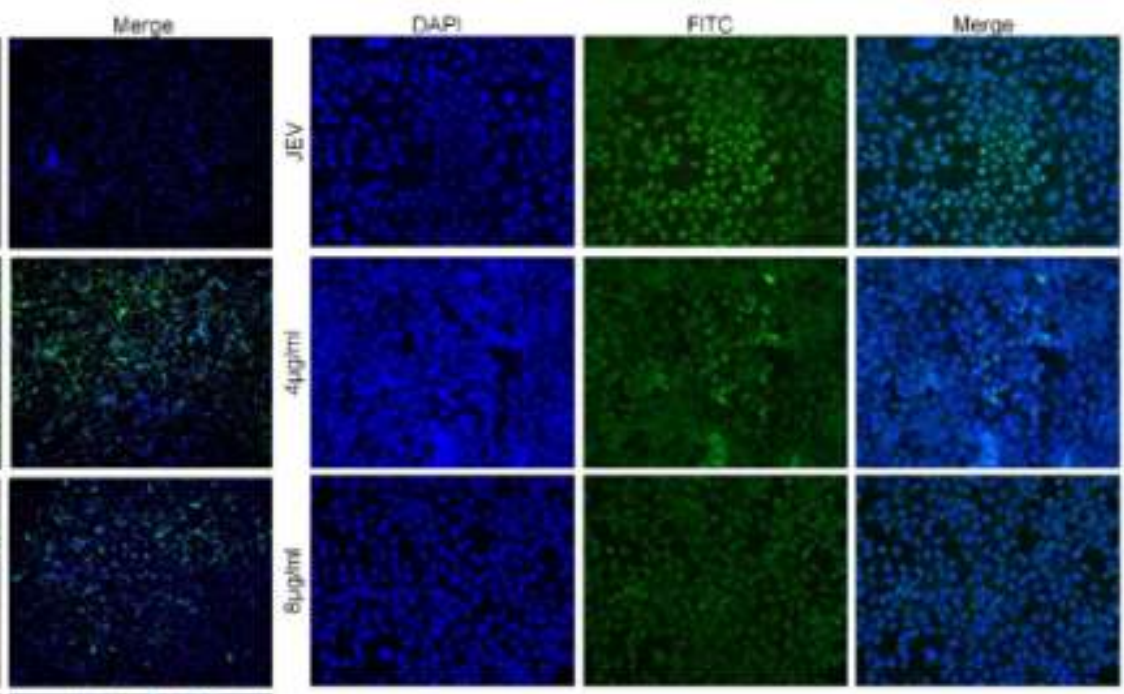

C

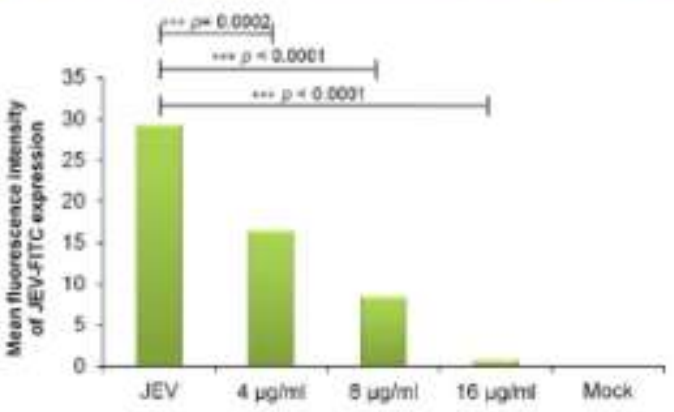

JEV
B200

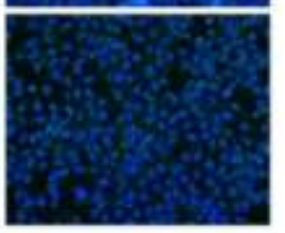

.

Figure 3: Antiviral activity of B200. (A) Immunofluorescence showing reduction in intracellular JEV levels in neuronal cells which were observed by FITC expression. (B)Immunofluorescence showing reduction in intracellular JEV levels in microglia cells which were observed by FITC expression.(C) Densitometric analysis showing reduction in mean fluorescent intensity of JEV-FITC expression where $2 \mu \mathrm{g} / \mathrm{ml}$ of B200 reduces the expression by 1.77 fold $(p=0.0002), 4 \mu \mathrm{g} / \mathrm{ml}$ reduces by 1.93 fold $(p<0.0001)$ and $6 \mu \mathrm{g} / \mathrm{ml}$ reduces by 11.19 fold $(p<0.0001)$.The data is expressed as the mean \pm standard error of mean where $p$ values are represented as the difference between JEV-infected cells and JEV-infected B200-treated cells. 
A

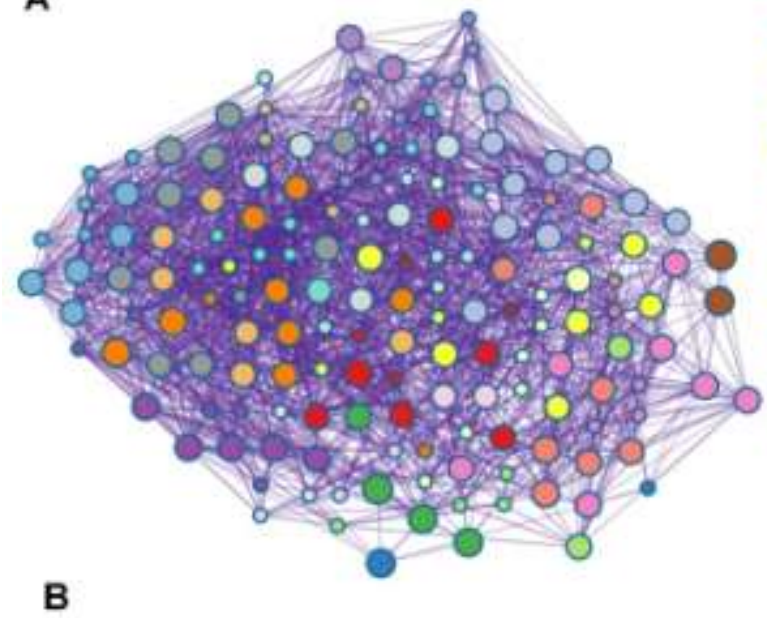

EEFR byoune kinase imhiter resstanch - Toxoplasmoes miepatis $\mathrm{B}$ evirinac Pathway tor Apoptom

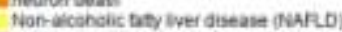

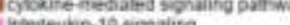
pontwo rojuason st neuran deah. Coiciectal cancer Teel acavabon STO 23 PATHinaY

ineuron apoptotic armcess mpeioid celil nomoctuz: NoO- ine rechetur noraling sathway posum irguason sf thepoesas negative reguation of phosichate motaboic process Actrason of BH3-onty probins

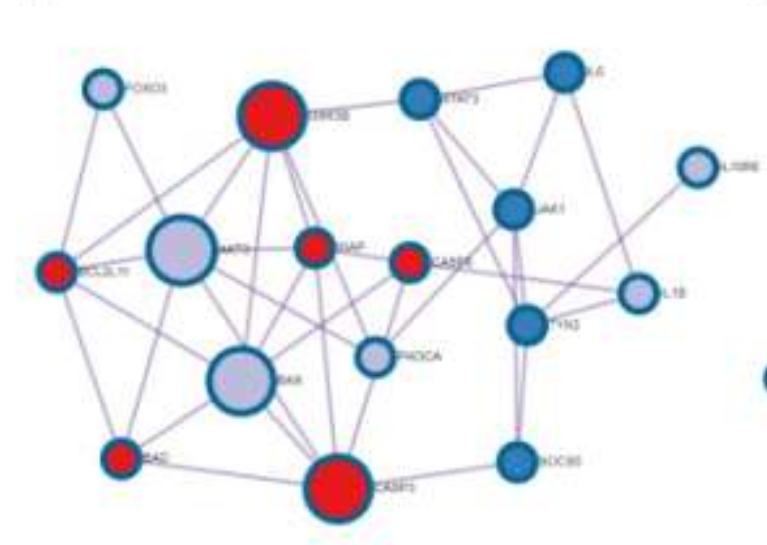

C
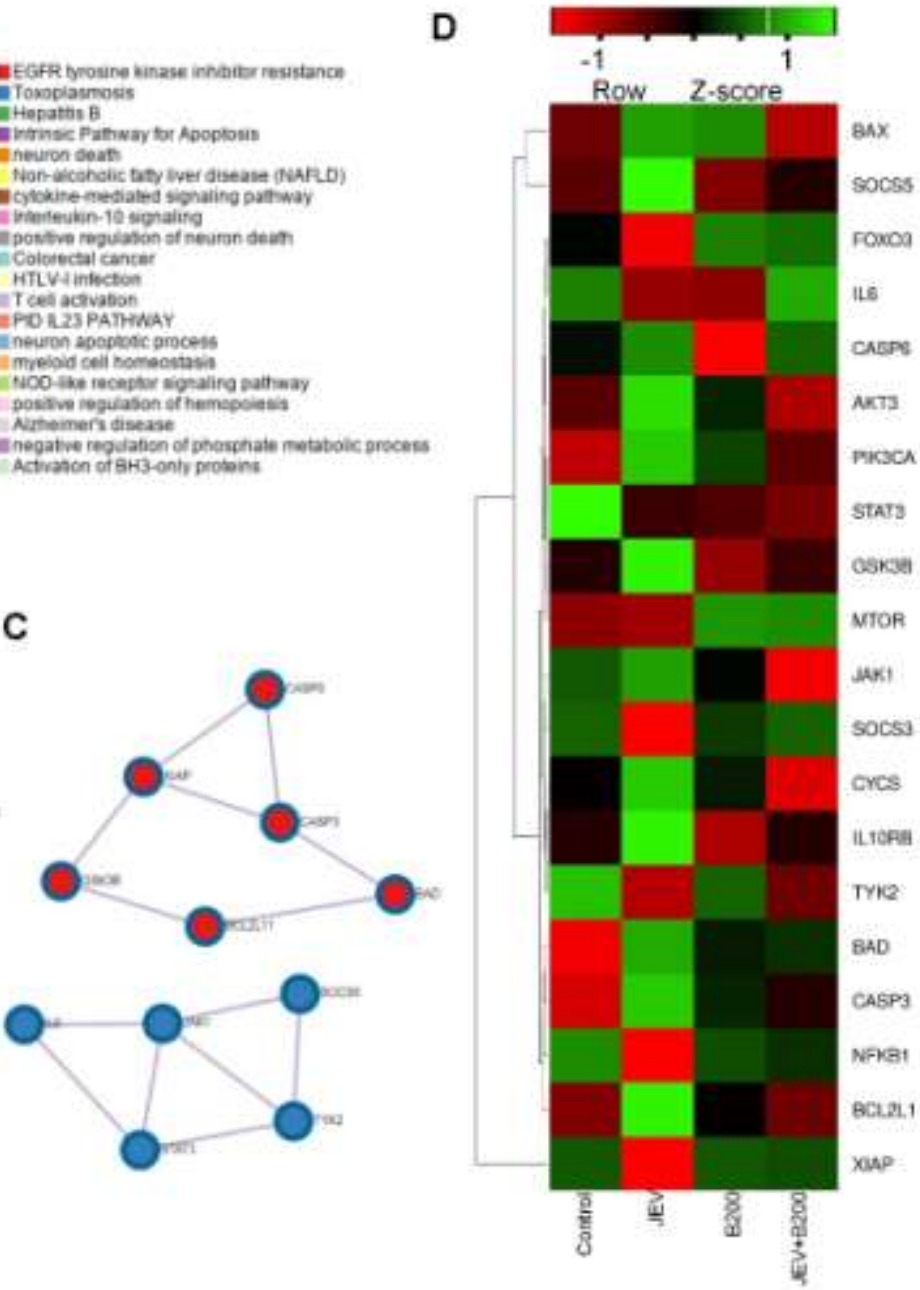

Figure 4: RNA seq and gene network analysis. (A) Gene enrichment and gene network analysis showing the involvement of several of the crucial pathways altered by B200 treatment including neuronal apoptotic process, interleukin 10 signaling, positive regulation of neuronal death. (B)Molecular complex detection (MCODE) algorithm was further applied to identify the key neighborhood genes. (C)Two sets of MCODE complexes were identified one comprises of CASP8, XIAP, CASP3, GSK3B, BAD, BCL2L1 1 and other comprises of JAK1, SOCS5, STAT3, TYK2 and IL6.(D)Heat map showing the levels of gene expression incontrol, JEV, B200 and JEV+B200 treated cells where expression of each gene was calculated as the ratio of the $\log _{2}$ expression value where red represents downregulated and green represents upregulated genes. 

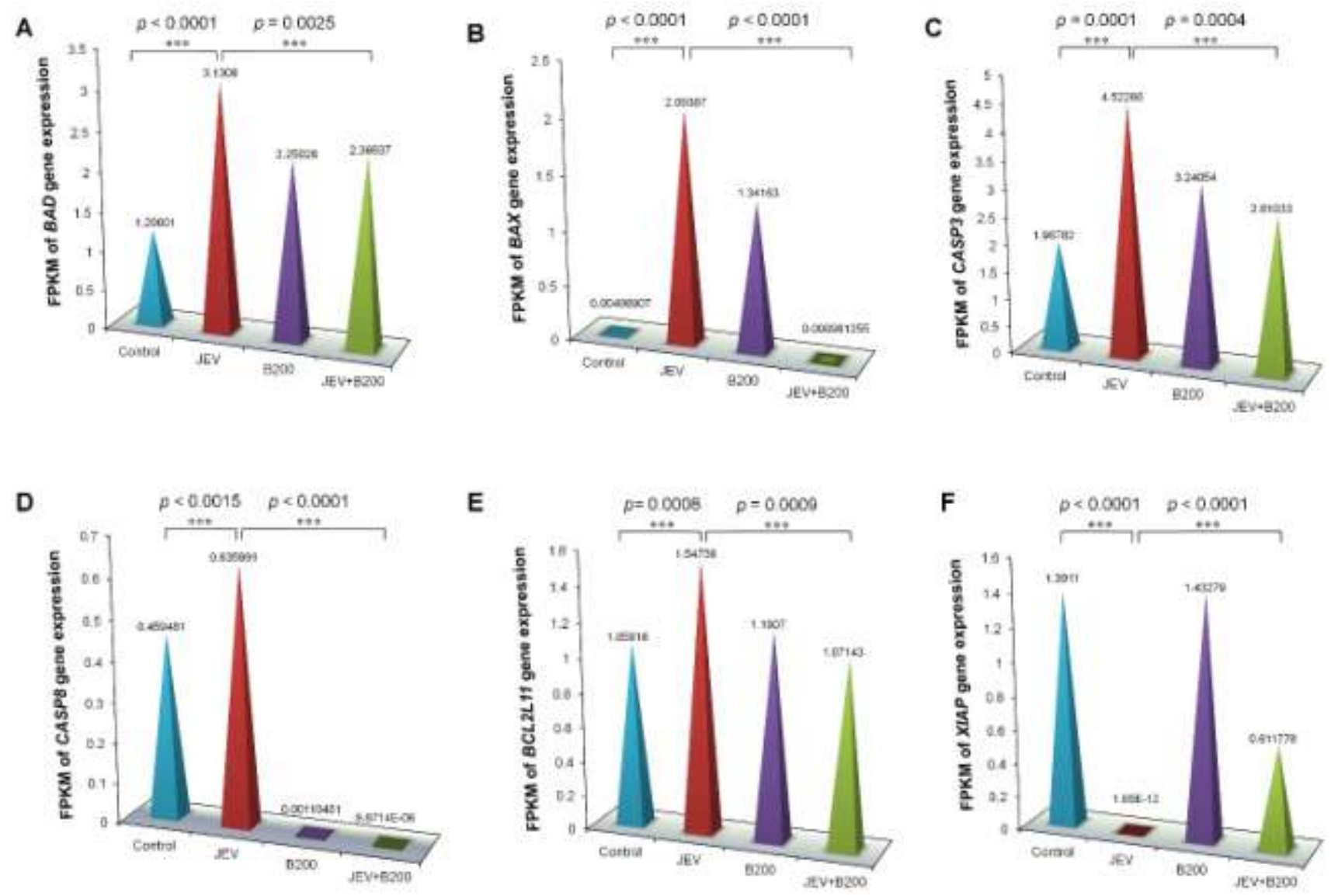

Figure 5:Downregulation of pro-apoptotic genes. Expression of apoptosis related genes was analyzed in JEV-infected and B200treated cells. (A) JEV-infected cells showed increase in $B A D$ expression by 2.59 fold $(p<0.0001)$ which was reduced by 1.32 fold ( $p=0.0025$ ) upon B200 treatment. (B) JEV-infected cells showed increase in $B A X$ expression by 419.69 fold $(p<0.0001)$ which was reduced by 2133.86 fold $(p<0.0001)$ upon B200 treatment. (C)JEV-infected cells showed increase in CASP3 expression by 2.29 fold $(p=0.0001)$ which was reduced by 1.60 fold $(p=0.0004)$ upon B200 treatment. (D) JEV-infected cells showed increase in CASP8 expression by 1.38 fold increase $(p=0.0015)$ which was reduced by 64427.63 fold $(p<0.0001)$ upon B200 treatment. (E)JEV-infected cells showed increase in BCL2L11 expression by 1.46 fold $(p=0.0008)$ which was reduced by 1.44 fold $(p=0.0009)$ upon B200 treatment. (F)JEV-infected cells showed reduction in XIAP expression by $8.43 \mathrm{E}+11$ fold $(p<0.0001)$ which was increased by $3.71 \mathrm{E}+11$ fold $(p<0.0001)$ upon B200 treatment. The data is expressed as the mean \pm standard error of mean where $p$ values are represented as the difference between JEV-infected cells and JEV-infected B200-treated cells. 
A
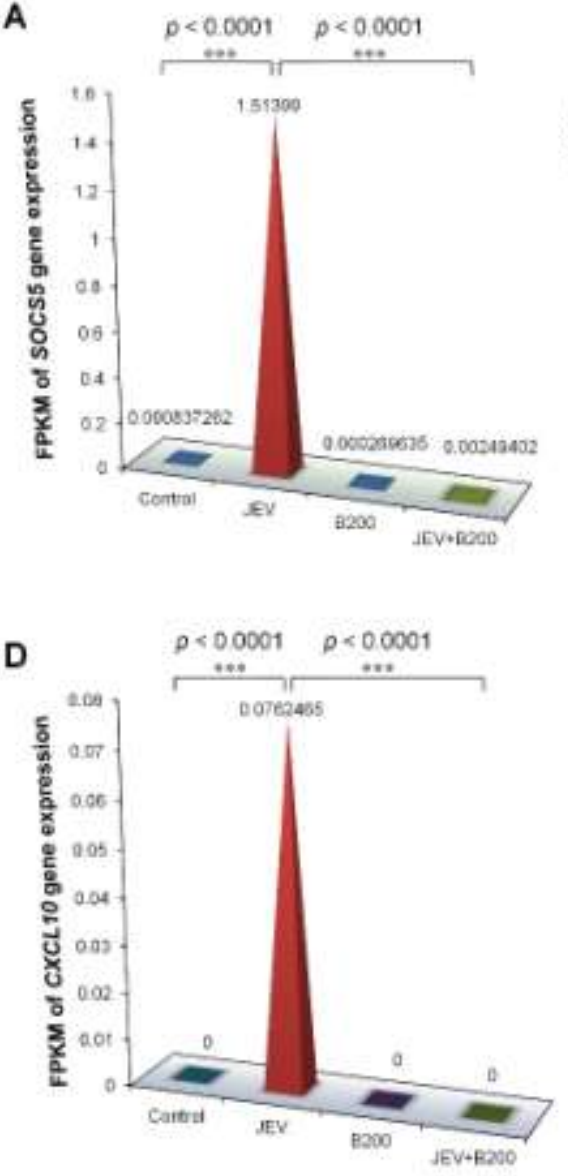
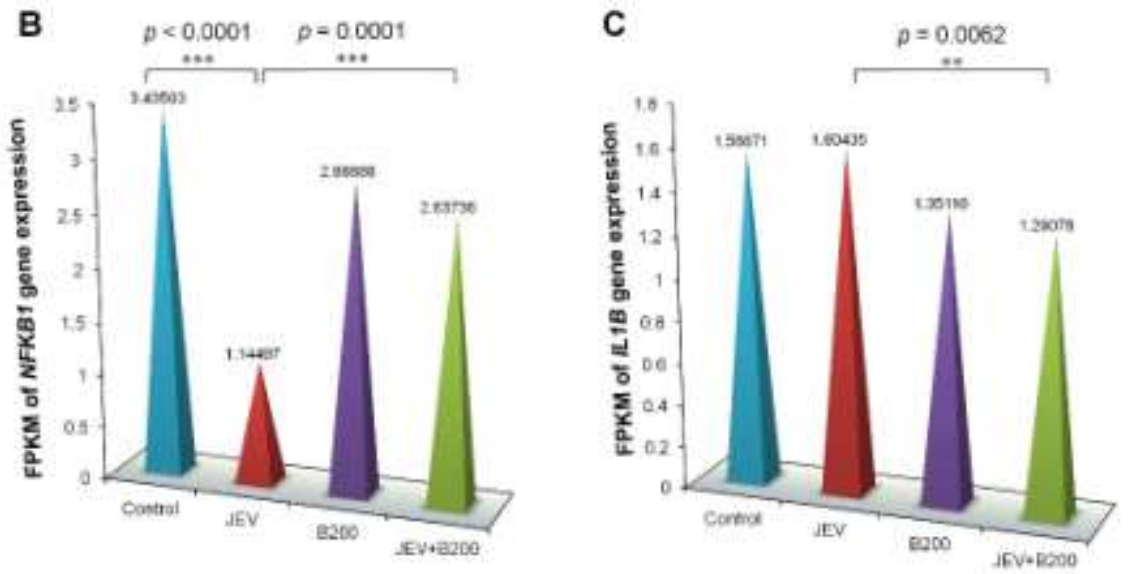

$\mathrm{E}$

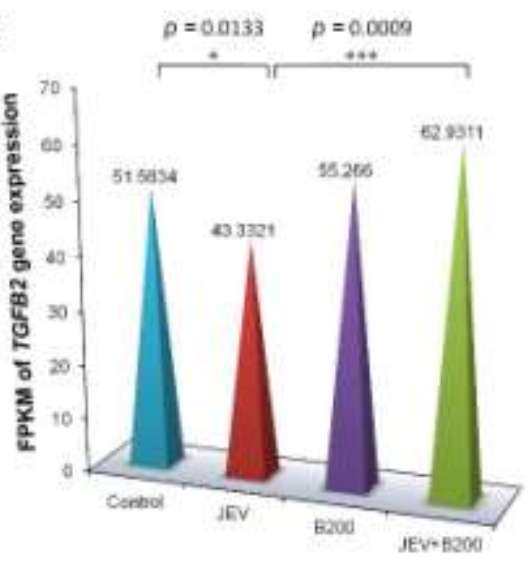

$\mathbf{F}$

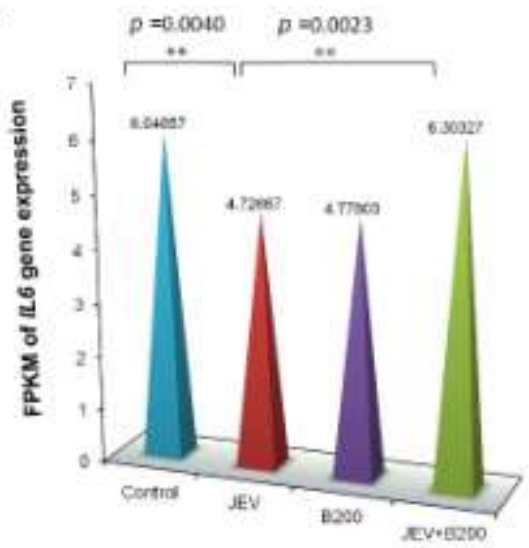

Figure 6:B200 mediated alteration of cytokines and inflammatory gene expression. Expression of inflammatory cytokine gene expression was analyzed in JEV-infected and B200-treated cells. (A) JEV-infected cells showed increase in SOCS5 expression by 1808.26 fold $(p<0.0001)$ which was reduced by 607.24 fold $(p<0.0001)$ upon B200 treatment. (B) JEV-infected cells showed reduction in NFKB1 expression by 3.00 fold $(p<0.0001)$ which was increased by $2.30(p=0.0001)$ upon B200 treatment. (C) JEV-infected cells showed increase in $I L 1 B$ expression by 1.02 ( $p=0.6114)$ which was reduced by fold 1.24 ( $p=0.0062$ )upon B200 treatment. (D)JEV-infected cells showed increase in CXCL10 expression by 76246.5 fold $(p<0.0001)$ which was reduced by 76246.5 fold ( $p<0.0001)$ upon B200 treatment. (E) JEV-infected cells showed reduction in TGFB2 expression by 1.18 fold $(p=0.0133)$ which was increased by 1.45 fold $(p=0.0009)$ upon B200 treatment. (F)JEV-infected cells showed reduction in IL6 expression by fold 1.27 fold $(p=0.0040)$ which was increased by 1.33 fold $(p=0.0023)$ upon B200 treatment.The data is expressed as the mean \pm standard error of mean where $p$ values are represented as the difference between JEV-infected cells and JEV-infected B200treated cells. 
A

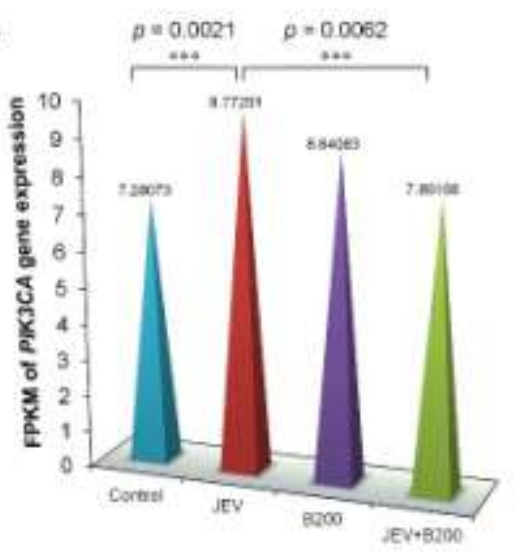

D

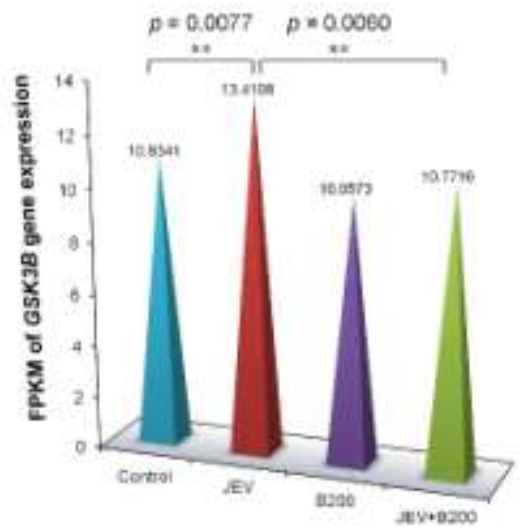

B



$E$

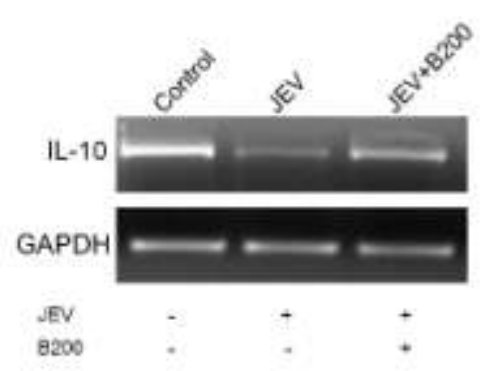

C

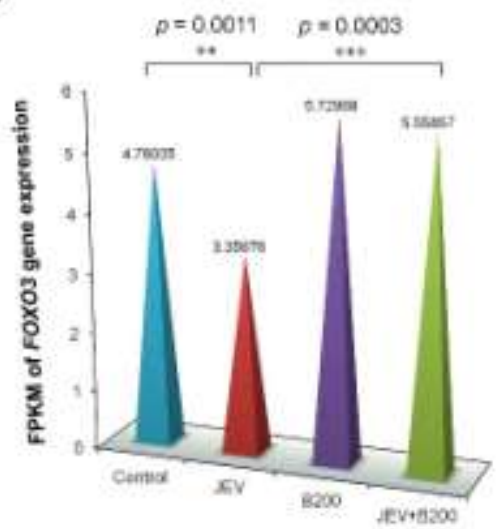

F

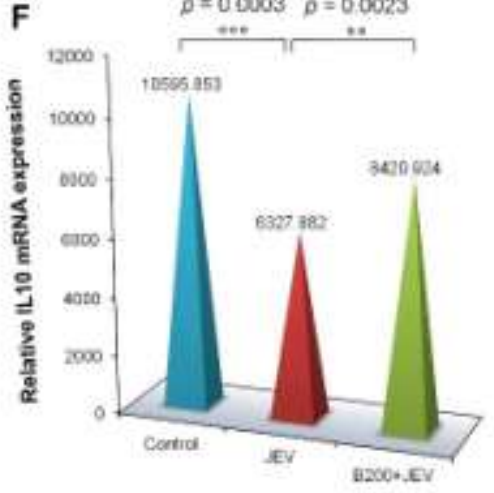

Figure 7:B200 mediated activation of PI3K/AKT pathway. Relative FPKM gene expression related to PI3K/AKT pathway was analyzed.(A) JEV-infected cells showed increase in PIK3CA expression by 1.34 fold $(p=0.0021)$ which was reduced by 1.24 fold ( $p=0.0062$ ) upon B200 treatment. (B) JEV-infected cells showed increase in AKT3 expression by 1.73 fold $(p=0.0002)$ which was reduced by 1.27 fold ( $p=0.0001$ ) upon B200 treatment. (C) JEV-infected cells showed reduction in FOXO3 expression by 1.42 fold ( $p=0.0011)$ which was increased by 1.65 fold $(p=0.0003)$ upon B200 treatment. (D)JEV infected cells showed increase in $G S K 3 B$ expression by1.22 fold ( $p=0.0077)$ which was reduced by1.24 fold $(p=0.0060)$ upon B200 treatment. (E) RT-PCR showing the relative mRNA expression of ILIO gene. (F)Densitometric analysis showing that JEV infection reduces the IL10expression by1.67 fold $(p=0.0003)$ which was increased by 1.33 fold $(p=0.0023)$ upon B200 treatment. The data is expressed as the mean \pm standard error of mean where $p$ values are represented as the difference between JEV-infected cells and JEV-infected B200treated cells. 


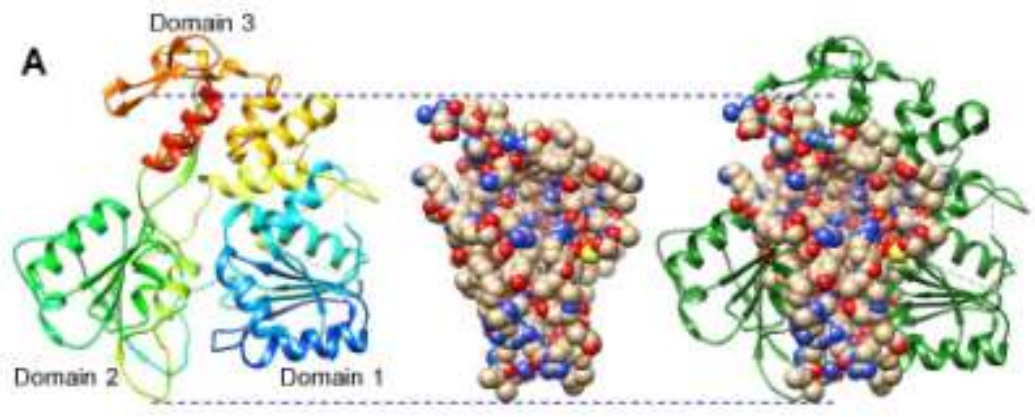

F
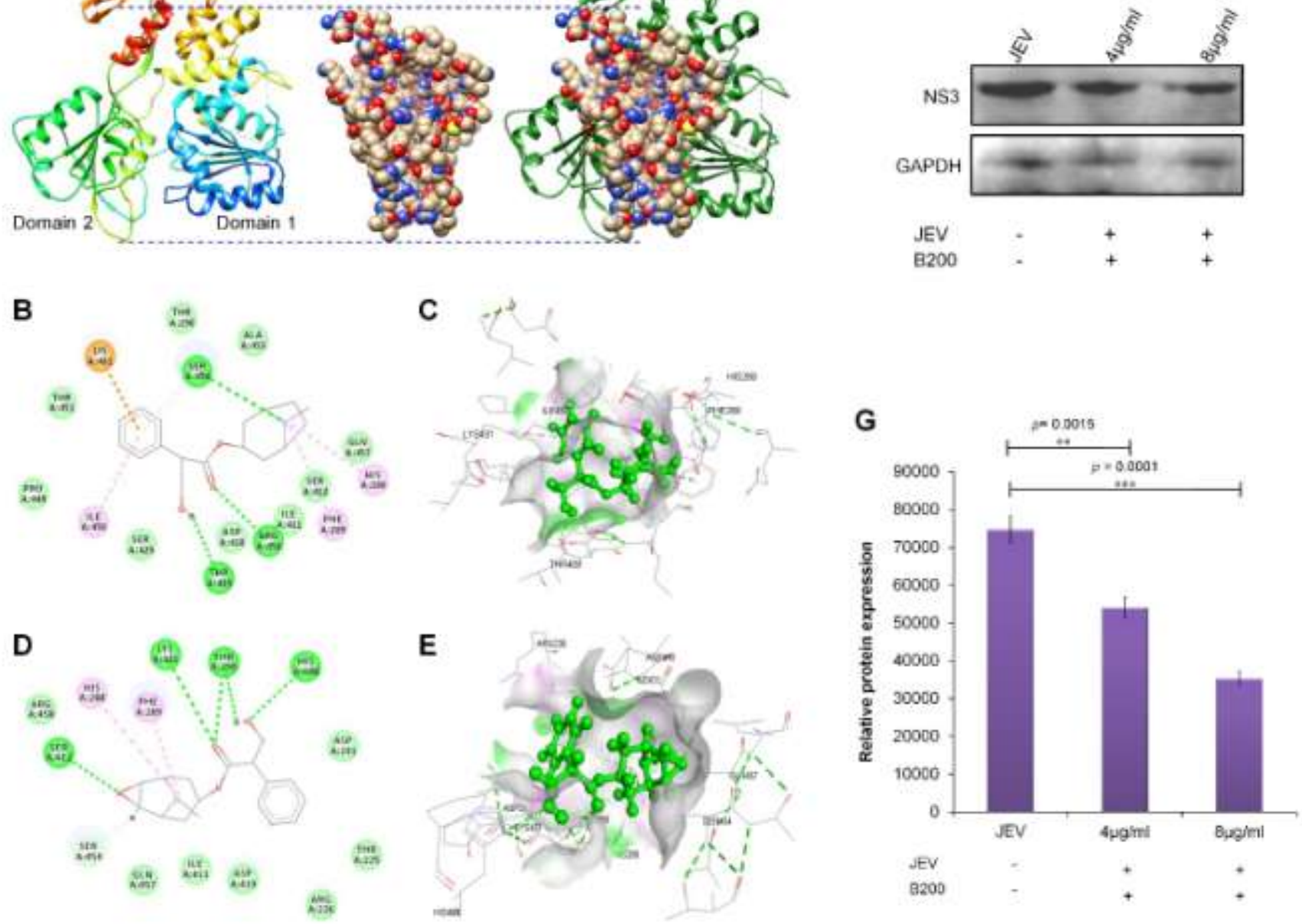

Figure 8: Reduction in B200 mediated NS3 protein expression. (A) Ribbon model representing the structure of JEV NS3 helicase/nucleoside triphosphatase with potential binding site of NS3 protein. (B) Two-dimensional and (C) three-dimensional view showing the interaction of atropine with His 288, Phe 289, Thr 409, Lys 431, Ile 450, Ser 454 and Arg 458 of NS3 protein. (D) Two-dimensional and (E) three-dimensional view showing the interaction of scopolamine with His 288, Phe 289, Thr 290, Ser 412, Lys 431 and His 488. (F)Western blotting was performed to observe the JEV NS3protein expression in B200 treated cells. (G)Densitometric analysis of the blot showing the relative protein expression. Treatment with $4 \mu \mathrm{g} / \mathrm{ml}$ and $6 \mu \mathrm{g} / \mathrm{ml} \mathrm{of} \mathrm{B200} \mathrm{reduces}$ the envelope protein expression by 1.37 fold $(p=0.0015)$ and 2.11 fold $(p=0.0001)$ respectively. The data is expressed as the mean \pm standard error of mean $(n=3)$ where $p$ values are represented as the difference between JEV-infected cells and JEV-infected B200-treated cells. 




Figure 9: Mechanism of B200 mediated antiviral activity and neuroprotection. B200 mediated reduction in microglia activation was marked by decrease in pro-inflammatory cytokines expression including IL1B and CXCL10 and therefore increases the neuronal cell survival. In addition, B200 treatment increases the anti-inflammatory cytokine expression especially IL-10 responsive genes and TGFB expression that further prevents the killing of neuronal cells via microglia activation. B200 treatment during JEV infection of microglia decreases the caspase dependent apoptosis and induces the NF- $\kappa \mathrm{B}$ translocation to the nucleus that further enhances the cell survival. Moreover, treatment was found to reduce the PI3K/AKT signaling and increases the FOXO3 expression which leads to cell survivability.Interestingly, we have found that belladonna alkaloids atropine and scopolamine both interacts with crucial residues of NS3 NTPase/helicase protein which resulted in degradation of NS3 protein. 
Table1. The drug likeness of B200*

\begin{tabular}{|c|c|c|}
\hline \multicolumn{3}{|c|}{ (A). Lipinski filter analysis } \\
\hline \multirow{2}{*}{ Lipinski filters } & \multicolumn{2}{|c|}{ Name of the alkaloids } \\
\hline & Atropine & Scopolamine \\
\hline Mass & 289.37 & 303.35 \\
\hline Hydrogen bond donor & 1 & 1 \\
\hline Hydrogen bond acceptors & 4 & 5 \\
\hline $\log P$ & 2.03 & 1.35 \\
\hline Molar Refractivity & 84.51 & 83.48 \\
\hline \multicolumn{3}{|c|}{ (B). admetSAR analysis } \\
\hline \multicolumn{3}{|c|}{ Absorption } \\
\hline Parameters & Atropine & Scopolamine \\
\hline Blood-Brain Barrier & $\mathrm{BBB}+$ & $\mathrm{BBB}+$ \\
\hline Human Intestinal absorption & HIA+ & $\mathrm{HIA}+$ \\
\hline Caco-2 Permeability & $\mathrm{Caco} 2+$ & $\mathrm{Caco} 2+$ \\
\hline P-glycoprotein Substrate & Substrate & Non substrate \\
\hline P-glycoprotein inhibitor & Non-inhibitor & Non-inhibitor \\
\hline \multicolumn{3}{|c|}{ Distribution } \\
\hline Sub cellular localization & Lysosome & Lysosome \\
\hline \multicolumn{3}{|c|}{ Metabolism } \\
\hline CYP450 2C9 Substrate & Non-substrate & Non-substrate \\
\hline CYP450 2C6 Substrate & Non-substrate & Non-substrate \\
\hline CYP450 2C9 Inhibitor & Non-inhibitor & Non-inhibitor \\
\hline CYP450 2C6 Inhibitor & Non-inhibitor & Non-inhibitor \\
\hline CYP450 3A4 Inhibitor & Non-inhibitor & Non-inhibitor \\
\hline \multicolumn{3}{|c|}{ Toxicity } \\
\hline AMES Toxicity & Non AMES toxic & Non AMES toxic \\
\hline Carcinogens & Non-carcinogens & Non-carcinogens \\
\hline Acute Oral Toxicity & III & III \\
\hline $\begin{array}{l}\text { Human Ether-a-go-go-Related } \\
\text { GeneInhibition (hERG) }\end{array}$ & Weak inhibitor & Weak inhibitor \\
\hline
\end{tabular}

*The drug likeness of B200 (atropine and scopolamine) was analyzed by Lipinski filter (A) admetSAR (B). 